\title{
ON A GENERALIZATION OF SOLOMON-TERAO FORMULA FOR SUBSPACE ARRANGEMENTS
}

\author{
DELPHINE POL
}

\begin{abstract}
We investigate in this paper a generalization of Solomon-Terao formula for central equidimensional subspace arrangements. We introduce generalized Solomon-Terao functions based on the Hilbert-Poincare series of the modules of multi-logarithmic forms and logarithmic multiresidues. We show that as in the case of hyperplane arrangements, these Solomon-Terao functions are polynomial. We then prove that if the Solomon-Terao polynomial of the modules of multiresidues satisfies a certain property, then this polynomial is related to the characteristic polynomial of the subspace arrangement. In particular, we prove that this generalized Solomon-Tearo formula holds for any line arrangement of any codimension.
\end{abstract}

\section{INTRODUCTION}

The characteristic polynomial of a subspace arrangement is an important invariant which carries information on the combinatorics and the topology of the arrangement and its complement. Over a finite field, it counts the number of points in the complement of the arrangement (see [Ath96]). For real $c$-arrangements, it is related to the Poincaré series and the Euler characteristic of the complement (see [Bjo94, Theorem 7.3.1, Theorem 8.2.1]). In the case of graphic hyperplane arrangements, it coincides with the chromatic polynomial, which is for example related to the "four colour theorem" (see $[$ OT92, 2.4]).

The characteristic polynomial also appears as a specialization of other polynomials attached to the arrangement. For example, it is related to Tutte polynomials (see for example [LNTY17] and the references within). In the case of hyperplane arrangements, the characteristic polynomial is given by a specialization of the Solomon-Terao polynomial associated with the modules of logarithmic vector fields or logarithmic differential forms ([ST87] and [OT92]). This relation is known as Solomon-Terao formula. An algebra called Solomon-Terao algebra which is related to the Solomon-Terao polynomial is introduced in the recent paper [AMMN18].

The purpose of this paper is to investigate a generalization of Solomon-Terao formula for equidimensional subspace arrangements, and in particular for line arrangements.

Multi-logarithmic differential forms and their residues along equidimensional analytic subspaces are introduced in [AT01] and [Ale14], which generalize the case of hypersurfaces (see [Sai80]). Several properties of logarithmic forms along hypersurfaces can be extended to complete intersections or equidimensional subspaces, in particular concerning freeness (see [Pol16a]).

Let $\mathscr{X}$ be an equidimensional subspace arrangement of codimension $k$ in $\mathbb{C}^{\ell}$. We set $S=$ $\mathbb{C}\left[x_{1}, \ldots, x_{\ell}\right]$ and for $q \in \mathbb{N}$ we set $\Omega^{q}$ the module of differential $q$-forms on $\mathbb{C}^{\ell}$. Let $\mathcal{I}_{\mathscr{X}} \subseteq S$ be the radical ideal of vanishing functions on $\mathscr{X}$. If $\mathscr{C}$ is an homogeneous reduced complete intersection defined by a regular sequence $\left(h_{1}, \ldots, h_{k}\right)$ such that $\mathscr{X} \subseteq \mathscr{C}$, we define the ideal $\mathcal{I}_{\mathscr{C}}=\left\langle h_{1}, \ldots, h_{k}\right\rangle \subseteq S$ and we set $h=h_{1} \cdots h_{k}$. For $q \in \mathbb{N}$, we define the module of multi-logarithmic differential $q$-forms

Date: March 3, 2022.

1991 Mathematics Subject Classification. 14N20 (Primary), 13D40, 13 N05.

Key words and phrases. subspace arrangements, Solomon-Terao formula, Hilbert-Poincaré series, logarithmic differential forms, logarithmic residues.

Research supported by a Japan Society for the Promotion of Science (JSPS) Postdoctoral Fellowship (Short-term) for North American and European Researchers. 
as:

$$
\Omega^{q}(\log \mathscr{X} / \mathscr{C})=\left\{\omega \in \frac{1}{h} \Omega^{q} ; \mathcal{I}_{\mathscr{X}} \omega \subseteq \frac{1}{h} \mathcal{I}_{\mathscr{C}} \Omega^{q} \text { and } \mathrm{d}\left(\mathcal{I}_{\mathscr{X}}\right) \wedge \omega \subseteq \frac{1}{h} \mathcal{I}_{\mathscr{C}} \Omega^{q+1}\right\} .
$$

We denote by $\mathcal{R}_{\mathscr{X}}^{q}$ the module of logarithmic multi-residues of $\Omega^{q+k}(\log \mathscr{X} / \mathscr{C})$, which does not depend on the choice of $\mathscr{C}$ (see definition 2.11).

Generalizing the Solomon-Terao polynomial, we define for any finite sequence of finitely generated graded $S$-modules $\left(M^{q}\right)_{0 \leqslant q \leqslant n}$ the $\Psi$-function as:

$$
\Psi\left(M^{\bullet}, x, t\right)=\sum_{q=0}^{n} \operatorname{Poin}\left(M^{q}, x\right)(t(1-x)-1)^{q},
$$

where Poin $\left(M^{q}, x\right)$ denotes the Hilbert-Poincaré series of $M^{q}$ (see definition 2.24). In particular, if $\mathscr{A}$ is an hyperplane arrangement and if $M^{\bullet}=\Omega^{\bullet}(\log \mathscr{A})$, it coincides with [OT92, Definition 4.130]. By Solomon-Terao formula, for any hyperplane arrangement $\mathscr{A}$, we have

$$
\Psi\left(\Omega^{\bullet}(\log \mathscr{A}), 1, t\right)=\chi(\mathscr{A}, t)
$$

where $\chi(\mathscr{A}, t)$ denotes the characteristic polynomial of $\mathscr{A}$ (see definition 2.28). In this paper, we will also consider $\Psi\left(\mathcal{R}_{\mathscr{X}}^{\bullet}, x, t\right)$ which is more convenient in the case of subspace arrangements.

Let $L(\mathscr{X})$ be the intersection lattice of $\mathscr{X}$ (see notation 2.26). For all $Y \in L(\mathscr{X}$ ), we denote by $\mathscr{X}_{Y}$ the subspace arrangement composed of the components of $\mathscr{X}$ which contain $Y$. The main results of this paper are (see theorem 4.2 and corollary 5.1):

Theorem. If $\mathscr{X}$ is a reduced equidimensional subspace arrangement in $\mathbb{C}^{\ell}$ and if for all $Y \in$ $L(\mathscr{X}) \backslash\{V\}$, the condition $\Psi\left(\mathcal{R}_{\mathscr{X}_{Y}}^{\bullet}, 1,1\right)=1$ is satisfied, then for all $Y \in L(\mathscr{X})$, we have

$$
\chi\left(\mathscr{X}_{Y}, t\right)=t^{\ell}-\Psi\left(\mathcal{R}_{\mathscr{X}_{Y}}, 1, t\right) .
$$

In particular, formula (1) holds for any line arrangement.

We also give an example of a subspace arrangement of dimension 2 which shows that formula (1) is not necessarily satisfied.

In section 2 , we first prove that for any equidimensional subspace arrangement $\mathscr{X}$ of codimension $k$ in $\mathbb{C}^{\ell}$, there exists a reduced subspace arrangement $\mathscr{C}$ of codimension $k$ which contains $\mathscr{X}$ and which is a complete intersection (see proposition 2.3). This property is then used to define the modules of multi-logarithmic differential forms $\Omega^{q}(\log \mathscr{X} / \mathscr{C})$ of $\mathscr{X}$ with respect to $\mathscr{C}$ (see definition 2.5), and to show that these modules are graded $S$-modules (see lemma 2.20). We also recall some properties of the modules of multi-logarithmic forms and multi-residues given in [Ale14] and [Pol16b]. We then define the $\Psi$-function associated with a family of finitely generated graded $S$-modules, and the characteristic polynomial of a subspace arrangement.

In section 3, we first prove that the $\Psi$-function associated with the modules of multi-logarithmic forms of an equidimensional subspace arrangement is a polynomial in $x, x^{-1}, t$ (see proposition 3.1). In proposition 3.10 we decompose the $\Psi$-function associated with the modules of multi-logarithmic forms with respect to the following exact sequence, which holds for all $q \in \mathbb{N}$ :

$$
0 \rightarrow \frac{1}{h} \mathcal{I}_{\mathscr{C}} \Omega^{q} \rightarrow \Omega^{q}(\log \mathscr{X} / \mathscr{C}) \rightarrow \mathcal{R}_{\mathscr{X}}^{q-k} \rightarrow 0
$$

The $\Psi$-function of $\frac{1}{h} \mathcal{I}_{\mathscr{C}} \Omega^{\bullet}$ can be computed thanks to Koszul complex (see proposition 3.10). We then explicitly compute the $\Psi$-function of the module of multi-residues in the case of complete intersection line arrangements thanks to [Pol16b, Théorème 6.1.29] (see proposition 3.13).

Section 4 is devoted to the main result of this paper. As suggested by the computation made in the case of complete intersection line arrangements, we introduce a function $\widetilde{\Psi}(\mathscr{X}, x, t)$ which coincides with the $\Psi$-function of the modules of multi-logarithmic forms if the codimension is odd (see notation 4.5), and which satisfies $\widetilde{\Psi}(\mathscr{X}, 1, t)=t^{\ell}-\Psi\left(\mathcal{R}_{\mathscr{X}}, 1, t\right)$. This function is used to prove our main theorem 4.2 . 
We then give several examples in section 5. We first consider the case of an arbitrary line arrangement $\mathscr{X}$. We deduce from the complete intersection case a generating family of the module of multi-residues of $\mathscr{X}$, which enables us to show that the $\Psi$-function of the module of multi-residues satisfies the required property, so that the generalized Solomon-Terao formula holds for any line arrangement. For higher dimensional subspaces, the generalized Solomon-Terao formula may not be satisfied. We give in subsection 5.2 an example of surface in $\mathbb{C}^{4}$ for which this formula is not satisfied. The question which remains open is to give characterizations of equidimensional subspace arrangements satisfying the Solomon-Terao formula.

Acknowledgments. The author is grateful to Masahiko Yoshinaga for pointing out this question, and for helpful discussions and comments and in particular for suggesting her to consider a kind of generic arrangement in proposition 2.3.

\section{Definitions}

We give in this section the definitions and some properties of multi-logarithmic forms and multiresidues along equidimensional subspace arrangements. These notions generalize the logarithmic differential forms along reduced hypersurfaces introduced by K. Saito in [Sai80]. These generalizations appear in [AT01], [Ale12] and [Ale14] in the case of germs of analytic subspaces. We will also recall results from [Pol16a] and [Pol16b].

\subsection{Multi-logarithmic forms and multi-residues.}

\subsubsection{Preliminary result. Let $\ell \in \mathbb{N}, \ell \geqslant 2$. We set $S=\mathbb{C}\left[x_{1}, \ldots, x_{\ell}\right]$.}

Definition 2.1. A subspace arrangement in $\mathbb{C}^{\ell}$ is a finite collection $\mathscr{X}=\left\{\mathscr{X}_{1}, \ldots, \mathscr{X}_{s}\right\}$ of affine subspaces of $\mathbb{C}^{\ell}$. We call $\mathscr{X}$ central if for all $i \in\{1, \ldots, s\}, 0 \in \mathscr{X}_{i}$. We call $\mathscr{X}$ equidimensional of codimension $k$ if for all $i \in\{1, \ldots, s\}$, the codimension of $\mathscr{X}_{i}$ in $\mathbb{C}^{\ell}$ is $k$.

In this paper, we will only consider central subspace arrangements, so that by subspace arrangement we will always mean central subspace arrangement. In addition, we will sometimes identify a subspace arrangement $\mathscr{X}=\left\{\mathscr{X}_{1}, \ldots, \mathscr{X}_{s}\right\}$ with $\bigcup_{i=1}^{s} \mathscr{X}_{i} \subseteq \mathbb{C}^{\ell}$.

Notation 2.2. Let $\mathscr{X} \subseteq \mathbb{C}^{\ell}$ be an equidimensional subspace arrangement. We denote by $\mathcal{I}_{\mathscr{X}} \subseteq S$ the ideal of vanishing functions on $\mathscr{X}$. In particular, $\mathcal{I}_{\mathscr{X}}$ is a radical and homogeneous ideal. We set $\mathcal{O}_{\mathscr{X}}=S / \mathcal{I}_{\mathscr{X}}$. We denote by $k$ the codimension of $\mathscr{X}$ in $\mathbb{C}^{\ell}$.

We will first need the following proposition:

Proposition 2.3. There exists a subspace arrangement $\mathscr{C}$ such that $\mathscr{C}$ is defined by an homogeneous regular sequence $\left(h_{1}, \ldots, h_{k}\right) \subseteq \mathcal{I}_{\mathscr{X}}$ and such that the ideal $\mathcal{I}_{\mathscr{C}}=\left\langle h_{1}, \ldots, h_{k}\right\rangle$ is radical.

Proof. The proof of this proposition consists on finding a kind of generic hyperplane arrangement up to codimension $k$. We will construct this hyperplane arrangement by induction.

Let $H_{1,1}, \ldots, H_{1, k}$ be hyperplanes of $\mathbb{C}^{\ell}$ such that $\bigcap_{i=1}^{k} H_{1, i}=\mathscr{X}_{1}$ and such that for all $i \in$ $\{1, \ldots, k\}$, for all $j \in\{2, \ldots, s\}, \mathscr{X}_{j} \not H_{1, i}$. We set for $j \in\{1, \ldots, k\}, \mathscr{A}_{1, j}=\left\{H_{1,1}, \ldots, H_{1, j}\right\}$.

Let $(i, j) \in\{1, \ldots, s\} \times\{1, \ldots, k\}$. We assume $(i, j) \neq(1,1)$. We set $(i, j)^{*}=(i, j-1)$ if $j \geqslant 2$ and $(i, j)^{*}=(i-1, k)$ if $j=1$.

For $(i, j) \in\{2, \ldots, s\} \times\{1, \ldots, k\}$ we define the hyperplane arrangements $\mathscr{A}_{i, j}$ inductively from $\mathscr{A}_{1, k}$ as follows.

Let $H_{i, j}$ be a hyperplane of $\mathbb{C}^{\ell}$ such that:

- $\mathscr{X}_{i} \subseteq H_{i, j}$,

- for all $n \in\{1, \ldots, s\}$, if $n \neq i, \mathscr{X}_{n} \nsubseteq H_{i, j}$,

- for all $1 \leqslant p \leqslant k$, for all $H_{1}, \ldots, H_{p} \in \mathscr{A}_{(i, j)^{*}}$, we have $H_{1} \cap \cdots \cap H_{p} \nsubseteq H_{i, j}$. 
Then we define $\mathscr{A}_{i, j}=\mathscr{A}_{(i, j)} \cup\left\{H_{i, j}\right\}$.

We thus obtain an hyperplane arrangement $\mathscr{A}_{s, k}$. For all $(i, j) \in\{1, \ldots, s\} \times\{1, \ldots, k\}$, let $\alpha_{i, j}$ be a reduced equation of the hyperplane $H_{i, j}$. For all $j \in\{1, \ldots, k\}$, we define $h_{j}=\prod_{i=1}^{s} \alpha_{i, j}$. Let us denote by $\mathscr{C}$ the variety defined by $\left(h_{1}, \ldots, h_{k}\right)$. We have:

$$
\mathscr{C}=\bigcup_{\left(i_{1}, \ldots, i_{k}\right) \in\{1, \ldots, s\}^{k}}\left(H_{i_{1}, 1} \cap \cdots \cap H_{i_{k}, k}\right) .
$$

Let us prove that $\mathscr{C}$ is a complete intersection containing $\mathscr{X}$ such that the ideal $\mathcal{I}_{\mathscr{C}}=\left\langle h_{1}, \ldots, h_{k}\right\rangle$ is radical, which is equivalent to proving the following three properties:

a) For all $\left(i_{1}, \ldots, i_{k}\right) \in\{1, \ldots, s\}^{k}$, the dimension of $H_{i_{1}, 1} \cap \cdots \cap H_{i_{k}, k}$ is $\ell-k$,

b) $\mathscr{X} \subseteq \mathscr{C}$,

c) For all $\left(i_{1}, \ldots, i_{k}\right) \in\{1, \ldots, s\}^{k}$, for all $\left(j_{1}, \ldots, j_{k}\right) \in\{1, \ldots, s\}^{k}$, if

$$
H_{i_{1}, 1} \cap \cdots \cap H_{i_{k}, k}=H_{j_{1}, 1} \cap \cdots \cap H_{j_{k}, k}
$$

then $\left(i_{1}, \ldots, i_{k}\right)=\left(j_{1}, \ldots, j_{k}\right)$.

Let us prove (a). It is sufficient to prove that for all $q \in\{1, \ldots, k-1\}$, for all $\left(i_{1}, \ldots, i_{q+1}\right) \in$ $\{1, \ldots, s\}^{q+1}$, we have:

$$
\operatorname{dim}\left(H_{i_{1}, 1} \cap \cdots \cap H_{i_{q}, q} \cap H_{i_{q+1}, q+1}\right)=\operatorname{dim}\left(H_{i_{1}, 1} \cap \cdots \cap H_{i_{q}, q}\right)-1 .
$$

Since all the hyperplanes contain the origin we have:

$$
\operatorname{dim}\left(H_{i_{1}, 1} \cap \cdots \cap H_{i_{q+1}, q+1}\right)-\operatorname{dim}\left(H_{i_{1}, 1} \cap \cdots \cap H_{i_{q}, q}\right) \in\{-1,0\} .
$$

Let us notice that $H_{i_{1}, 1}, \ldots, H_{i_{q}, q} \in \mathscr{A}_{\left(i_{q+1}, q+1\right)^{*}}$, so that by assumption, $H_{i_{1}, 1} \cap \cdots \cap H_{i_{q}, q} \nsubseteq$ $H_{i_{q+1}, q+1}$. Therefore, the two dimensions in (2) cannot be equal. Hence the result.

Let us prove (b). Let $i \in\{1, \ldots, s\}$. By assumption, for all $j \in\{1, \ldots, k\}, \mathscr{X}_{i} \subseteq H_{i, j}$. Therefore, $\mathscr{X}_{i} \subseteq H_{i, 1} \cap \cdots \cap H_{i, k} \subseteq \mathscr{C}$. More precisely, from (a), $\operatorname{dim}\left(H_{i, 1} \cap \cdots \cap H_{i, k}\right)=\ell-k=\operatorname{dim}\left(\mathscr{X}_{i}\right)$, so that we have $H_{i, 1} \cap \cdots \cap H_{i, k}=\mathscr{X}_{i}$ since both are vector subspaces of the same dimension.

It remains to prove (c), which will show that the complete intersection $\mathscr{C}$ is reduced. One can notice that since $\mathcal{O}_{\mathscr{C}}$ is a complete intersection ring, there is no embedding prime. Let us assume that

$$
H_{i_{1}, 1} \cap \cdots \cap H_{i_{k}, k}=H_{j_{1}, 1} \cap \cdots \cap H_{j_{k}, k} .
$$

If $i_{k} \neq j_{k}$, then we may assume that $i_{k}<j_{k}$. Then $H_{i_{1}, 1}, \ldots, H_{i_{k}, k} \in \mathscr{A}_{\left(j_{k}, k\right)^{*}}$, so that $H_{i_{1}, 1} \cap \cdots \cap$ $H_{i_{k}, k} \not \subset H_{j_{k}, k}$, which is contradictory.

Thus, $i_{k}=j_{k}$. Let us prove that $H_{i_{1}, 1} \cap \cdots \cap H_{i_{k-1}, k-1}=H_{j_{1}, 1} \cap \ldots H_{j_{k-1}, k-1}$. We have:

$$
\operatorname{dim}\left(\left(H_{i_{1}, 1} \cap \cdots \cap H_{i_{k-1}, k-1} \cap H_{j_{1}} \cap \ldots H_{j_{k-1}, k-1}\right) \cap H_{i_{k}, k}\right)=\ell-k .
$$

Thus, $\delta:=\operatorname{dim}\left(H_{i_{1}, 1} \cap \cdots \cap H_{i_{k-1}, k-1} \cap H_{j_{1}} \cap \cdots \cap H_{j_{k-1}, k-1}\right) \in\{\ell-k, \ell-k+1\}$.

Since $\operatorname{dim}\left(H_{i_{1}, 1} \cap \cdots \cap H_{i_{k-1}, k-1}\right)=\ell-k+1$, if $\delta=\ell-k$, there exists $n \in\{1, \ldots, k-1\}$ such that $\operatorname{dim}\left(H_{i_{1}, 1} \cap \cdots \cap H_{i_{k-1}, k-1} \cap H_{j_{n}, n}\right)=\ell-k=\operatorname{dim}\left(H_{i_{1}, 1} \cap \cdots \cap H_{i_{k-1}, k-1} \cap H_{j_{n}, n} \cap H_{i_{k}, k}\right)$. However, $H_{i_{1}, 1}, \ldots, H_{i_{k-1}, k-1}, H_{j_{n}, n} \in \mathscr{A}_{\left(i_{k}, k\right)^{*}}$, so that $H_{i_{1}, 1} \cap \cdots \cap H_{i_{k-1}, k-1} \cap H_{j_{n}, n} \not H_{i_{k}, k}$, which is a contraction. Thus, $\delta=\ell-k+1$. Since $\operatorname{dim}\left(H_{i_{1}, 1} \cap \cdots \cap H_{i_{k-1}, k-1}\right)=\operatorname{dim}\left(H_{j_{1}, 1} \cap \cdots \cap H_{j_{k-1}, k-1}\right)=$ $\ell-k+1$, we have:

$$
H_{i_{1}, 1} \cap \cdots \cap H_{i_{k-1}, k-1}=H_{j_{1}, 1} \cap \cdots \cap H_{j_{k-1}, k-1} .
$$

Then, in a completely similar way, one can prove by induction that $\left(i_{1}, \ldots, i_{k}\right)=\left(j_{1}, \ldots, j_{k}\right)$.

Therefore, $\mathscr{C}$ is a reduced complete intersection subspace arrangement of codimension $k$ containing $\mathscr{X}$.

Remark 2.4. This result is not implied by [Pol16b, Proposition 4.2.1], where we prove only that for any germ of reduced equidimensional subspace $\mathscr{X} \subseteq\left(\mathbb{C}^{\ell}, 0\right)$, there exists a germ of reduced complete intersection $\mathscr{C}$ containing $\mathscr{X}$ of the same dimension, but where $\mathscr{C}$ may not be homogeneous even if $\mathscr{X}$ is homogeneous. 
2.1.2. Definitions. We fix a reduced complete intersection $\mathscr{C}$ satisfying proposition 2.3 . We set $h=h_{1} \cdots h_{k}$. We then define the module of multi-logarithmic forms as follows:

Definition 2.5 (see [Ale14, Definition 10.1]). Let $q \in \mathbb{N}$. The module of multi-logarithmic q-forms along $\mathscr{X}$ with respect to $\mathscr{C}$ is defined by:

$$
\Omega^{q}(\log \mathscr{X} / \mathscr{C})=\left\{\omega \in \frac{1}{h} \Omega^{q} ; \mathcal{I}_{\mathscr{X}} \omega \subseteq \frac{1}{h} \mathcal{I}_{\mathscr{C}} \Omega^{q} \text { and } \mathrm{d}\left(\mathcal{I}_{\mathscr{X}}\right) \wedge \omega \subseteq \frac{1}{h} \mathcal{I}_{\mathscr{C}} \Omega^{q+1}\right\} .
$$

Remark 2.6. If $\mathscr{C}$ is a reduced complete intersection, we set

$$
\Omega^{q}(\log \mathscr{C}):=\Omega^{q}(\log \mathscr{C} / \mathscr{C})=\left\{\omega \in \frac{1}{h} \Omega^{q} ; \mathrm{d}\left(\mathcal{I}_{\mathscr{C}}\right) \wedge \omega \subseteq \frac{1}{h} \mathcal{I}_{\mathscr{C}} \Omega^{q}\right\} .
$$

Remark 2.7 (see $[$ Ale14, §10]). We have the following inclusion:

$$
\Omega^{q}(\log \mathscr{X} / \mathscr{C}) \subseteq \Omega^{q}(\log \mathscr{C}) .
$$

Let us denote $\mathscr{C}=\mathscr{X}_{1} \cup \cdots \cup \mathscr{X}_{s} \cup \mathscr{Y}_{1} \cup \cdots \cup \mathscr{Y}_{r}$, where $\mathscr{Y}_{1}, \ldots, \mathscr{Y}_{r}$ are the irreducible components of $\mathscr{C}$ which are not in $\mathscr{X}$. We set $\mathcal{O}_{\mathscr{C}}=S / \mathcal{I}_{\mathscr{C}}$.

For a ring $R$, we denote by $\operatorname{Frac}(R)$ the total ring of fractions of $R$.

Definition 2.8 ([Ker84, 1.3]). Let $c_{\mathscr{X} / \mathscr{C}} \in \Omega^{k}$. We say that $c_{\mathscr{X} / \mathscr{C}}$ is a fundamental form of $\mathscr{X}$ if we have:

$$
\overline{c_{\mathscr{X} / \mathscr{C}}}=\overline{\beta_{\mathscr{X} / \mathscr{C}} \mathrm{d} h_{1} \wedge \cdots \wedge \mathrm{d} h_{k}} \in \frac{\Omega^{k}}{\mathcal{I}_{\mathscr{C}} \Omega^{k}}
$$

where $\beta_{\mathscr{X} / \mathscr{C}} \in \operatorname{Frac}\left(\mathcal{O}_{\mathscr{C}}\right)$ satisfies for all $i \in\{1, \ldots, s\}, \beta_{\mathscr{X}} /\left.\mathscr{C}\right|_{\mathscr{X}_{i}}=1$ and for all $j \in\{1, \ldots, r\}$, $\left.\beta_{\mathscr{X} / \mathscr{C}}\right|_{\mathscr{Y} i}=0$, and $\overline{c_{\mathscr{X} / \mathscr{C}}}$ denotes the class of $c_{\mathscr{X} / \mathscr{C}}$ in $\frac{\Omega^{k}}{\mathcal{I}_{\mathscr{C}} \Omega^{k}}$.

Remark 2.9. In particular, if $\mathscr{X}=\mathscr{C}$ is a reduced complete intersection, then one can take $c_{\mathscr{C}} / \mathscr{C}=$ $\mathrm{d} h_{1} \wedge \cdots \wedge \mathrm{d} h_{k}$.

In order to introduce the module of logarithmic multi-residues, we need the following theorem, which gives a characterization of multi-logarithmic forms and generalizes [Sai80, (1.1)].

Theorem 2.10 (see [Ale12, §3, Theorem 1] and [Pol16b, Proposition 4.2.6]). Let $q \in \mathbb{N}$. Let $\omega \in \frac{1}{h} \Omega^{q}$. Then $\omega \in \Omega^{q}(\log \mathscr{X} / \mathscr{C})$ if and only if there exist $g \in S$ inducing a non zero divisor ${ }^{1}$ in $\mathcal{O}_{\mathscr{C}}, \xi \in \Omega^{q-k}$ and $\eta \in \frac{1}{h} \mathcal{I}_{\mathscr{C}} \Omega^{q}$ such that

$$
g \omega=\frac{c_{\mathscr{X}} / \mathscr{C}}{h} \wedge \xi+\eta
$$

Definition 2.11. Let $q \in \mathbb{N}$. Let $\omega \in \Omega^{q}(\log \mathscr{X} / \mathscr{C})$. With the notations of the theorem, the multi-residue of $\omega$ is:

$$
\operatorname{res} \mathscr{X} / \mathscr{C}(\omega)=\frac{\xi}{g} \in \Omega_{\mathscr{X}}^{q-k} \otimes \operatorname{Frac}\left(\mathcal{O}_{\mathscr{X}}\right)
$$

where for $p \in \mathbb{N}, \Omega_{\mathscr{X}}^{p}=\frac{\Omega^{p}}{\mathcal{I}_{\mathscr{X}} \Omega^{p}+\mathrm{d}\left(\mathcal{I}_{\mathscr{X}}\right) \wedge \Omega^{p-1}}$

Notation 2.12. For $q \in \mathbb{N}$, we denote $\mathcal{R}_{\mathscr{X}}^{q}=\operatorname{res}_{\mathscr{X} / \mathscr{C}}\left(\Omega^{q+k}(\log \mathscr{X} / \mathscr{C})\right)$.

Remark 2.13. For all $q \in \mathbb{N}$, the module $\mathcal{R}_{\mathscr{X}}^{q}$ depends only on $\mathscr{X}$, contrary to the modules $\Omega^{q}(\log \mathscr{X} / \mathscr{C})$ which depend on the choice of the complete intersection $\mathscr{C}$ (see [Ale14, 110$]$ and [Pol16b, Proposition 4.1.5 and 4.1.13]).

Remark 2.14. For all $q \in \mathbb{N}$, the module of multi-residues $\mathcal{R}_{\mathscr{X}}^{q}$ is isomorphic to the module of regular meromorphic forms $\omega_{\mathscr{X}}^{q}$ (see $[$ Ale14, $\S 10]$ ). The module $\omega_{\mathscr{X}}^{\ell-k}$ is the dualizing module given by $\omega_{\mathscr{X}}^{\ell-k}=\operatorname{Ext}_{S}^{k}\left(\mathcal{O}_{\mathscr{X}}, \Omega^{\ell}\right)$ and for all $q, \omega_{\mathscr{X}}^{q}=\operatorname{Hom}_{\mathcal{O}_{\mathscr{X}}}\left(\Omega_{\mathscr{X}}^{\ell-k-q}, \omega_{\mathscr{X}}^{\ell-k}\right)$.

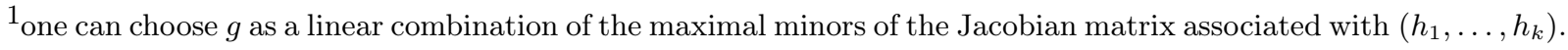


Proposition 2.15 ([Ale14, Theorem 10.2] and [Pol16b, Corollaire 4.1.9]). For all $q \in \mathbb{N}$, we have the following exact sequence:

$$
0 \rightarrow \frac{1}{h} \mathcal{I}_{\mathscr{C}} \Omega^{q} \rightarrow \Omega^{q}(\log \mathscr{X} / \mathscr{C}) \rightarrow \mathcal{R}_{\mathscr{X}}^{q-k} \rightarrow 0
$$

Remark 2.16. For all $q<k, \Omega^{q}(\log \mathscr{X} / \mathscr{C})=\frac{1}{h} \mathcal{I}_{\mathscr{C}} \Omega^{q}$.

Remark 2.17. By remark 2.7, we have $\Omega^{q}(\log \mathscr{X} / \mathscr{C}) \subseteq \Omega^{q}(\log \mathscr{C})$. If $\omega \in \Omega^{q}(\log \mathscr{X} / \mathscr{C})$, we also have $\operatorname{res} \mathscr{X} / \mathscr{C}(\omega)=\left.\operatorname{res}_{\mathscr{C}}(\omega)\right|_{\mathscr{X}} \in \Omega_{\mathscr{X}}^{q-k} \otimes \operatorname{Frac}\left(\mathcal{O}_{\mathscr{X}}\right)$. In addition, we have an inclusion $\mathcal{R}_{\mathscr{X}}^{q} \hookrightarrow \mathcal{R}_{\mathscr{C}}^{q}$ which is defined as follows: for any $\omega \in \Omega^{q}(\log \mathscr{X} / \mathscr{C})$, res $\mathscr{X} / \mathscr{C}(\omega) \in \mathcal{R}_{\mathscr{X}}^{q} \mapsto \operatorname{res}_{\mathscr{C}}(\omega) \in \mathcal{R}_{\mathscr{C}}^{q}$. Since the kernel of $\operatorname{res} \mathscr{X} / \mathscr{C}$ is $\frac{1}{h} \mathcal{I}_{\mathscr{C}} \Omega^{q}$ which is also the kernel of res $\mathscr{C}$, the previous map is well defined and is an inclusion.

\subsection{Poincaré polynomials, $\Psi$-functions and characteristic polynomial.}

2.2.1. Graduation. We keep the same notations as before. In order to consider the Hilbert-Poincaré series of $\Omega^{\bullet}(\log \mathscr{X} / \mathscr{C})$ and $\mathcal{R}_{\mathscr{X}}^{\bullet}$, we first need to introduce a graduation on these modules.

Notation 2.18. For $q \in\{1, \ldots, \ell\}$ and $I=\left\{i_{1}, \ldots, i_{q}\right\} \subseteq\{1, \ldots, \ell\}$, we set $\mathrm{d} x_{I}=\mathrm{d} x_{i_{1}} \wedge \cdots \wedge \mathrm{d} x_{i_{q}}$. We denote by $|I|$ the cardinality of the set $I$.

Definition 2.19. A form $\alpha=\sum_{|I|=q} a_{I} \mathrm{~d} x_{I} \in \Omega^{q}$ is called homogeneous of polynomial degree $p$ if for all $I, a_{I}$ is an homogeneous polynomial of degree $p$.

Lemma 2.20. The modules $\Omega^{q}(\log \mathscr{X} / \mathscr{C})$ are finitely generated graded $S$-modules with the graduation induced by the polynomial degree on $\Omega^{q}$.

Proof. Let $\left(f_{1}, \ldots, f_{r}\right)$ be homogeneous polynomials such that $\left\langle f_{1}, \ldots, f_{r}\right\rangle=\mathcal{I}_{\mathscr{X}}$. For all $i \in$ $\{1, \ldots, r\}$, we set $\delta_{i}=\operatorname{deg}\left(f_{i}\right)$.

The module $\Omega^{q}(\log \mathscr{X} / \mathscr{C})$ can be seen as the kernel of the morphism of graded modules:

$$
\frac{1}{h} \Omega^{q} \stackrel{\phi}{\rightarrow} \bigoplus_{i=1}^{r} \frac{\Omega^{q}}{\mathcal{I}_{\mathscr{C}} \Omega^{q}}\left(\delta_{i}\right) \oplus \bigoplus_{i=1}^{r} \frac{\Omega^{q+1}}{\mathcal{I}_{\mathscr{C}} \Omega^{q+1}}\left(\delta_{i}-1\right)
$$

defined by $\phi(\omega)=\left(\left(f_{i} h \omega\right)_{i \in\{1, \ldots, r\}},\left(\mathrm{d} f_{i} \wedge h \omega\right)_{i \in\{1, \ldots, r\}}\right)$. Therefore, $\Omega^{q}(\log \mathscr{X} / \mathscr{C})$ is a graded $S$ module.

Notation 2.21. For all $i \in\{1, \ldots, k\}$, we denote by $d_{i}$ the degree of the homogeneous polynomial $h_{i}$, and we set $d=\sum_{i=1}^{k} d_{i}=\operatorname{deg}(h)$.

For all $K \subseteq\{1, \ldots, \ell\}$ with $|K|=k$, we set $J_{K}$ the $(k \times k)$-minor of the Jacobian matrix of $\left(h_{1}, \ldots, h_{k}\right)$ relative to the set $K$. In particular, $\mathrm{d} h_{1} \wedge \cdots \wedge \mathrm{d} h_{k}=\sum_{|K|=k} J_{K} \mathrm{~d} x_{K}$. In addition, for all $K \subseteq\{1, \ldots, \ell\}$ with $|K|=k$, we have $J_{K}=0$ or $\operatorname{deg}\left(J_{K}\right)=d-k$. Thus, $\operatorname{deg}\left(\operatorname{d} h_{1} \wedge \cdots \wedge \mathrm{d} h_{k}\right)=d-k$.

Proposition 2.22. The map res $\mathscr{X} / \mathscr{C}$ is homogeneous of degree $k$.

Proof. Let $\omega \in \Omega^{q}(\log \mathscr{X} / \mathscr{C}) \subseteq \Omega^{q}(\log \mathscr{C})$ be an homogeneous multi-logarithmic form. Since $\omega$ is a multi-logarithmic form along $\mathscr{C}$, there exist $g, \xi, \eta$ as in theorem 2.10 such that

$$
g \omega=\frac{\mathrm{d} h_{1} \wedge \cdots \wedge \mathrm{d} h_{k}}{h} \wedge \xi+\eta .
$$

In particular, since $g$ can be chosen as a $\mathbb{C}$-linear combination of the maximal minors of the Jacobian matrix, one can assume that $g$ is homogeneous of degree $d-k$, so that $g \omega$ is an homogeneous form. Therefore, we may assume that $\xi$ and $\eta$ are homogeneous elements respectively of degree $\operatorname{deg}(\omega)+d$ and $d-k+\operatorname{deg}(\omega)$. Then $\operatorname{res}_{\mathscr{X}} / \mathscr{C}(\omega)=\frac{\xi}{g}$ is homogeneous of degree $\operatorname{deg}(\omega)+k$. Hence the result. 
Remark 2.23. As mentioned in remark 2.14, the modules of multi-residues are isomorphic to the modules of regular meromorphic forms $\omega_{\mathscr{X}}^{q}$. Using [Ker84, (1.2)], one can prove that the isomorphism $\mathcal{R}_{\mathscr{X}}^{q} \rightarrow \omega_{\mathscr{X}}^{q}$ is homogeneous of degree $k$. Indeed, by [Ker84, (1.2)], we have:

$$
\begin{aligned}
\omega_{\mathscr{X}}^{q}= & \left\{\left[\begin{array}{c}
\alpha \\
f_{1}, \ldots, f_{k}
\end{array}\right] ; \alpha \in \Omega^{q+k},\left(f_{1}, \ldots, f_{k}\right) \subseteq \mathcal{I}_{\mathscr{X}}\right. \text { a regular sequence, } \\
& \left.\mathcal{I}_{\mathscr{X}} \alpha \subseteq\left(f_{1}, \ldots, f_{k}\right) \Omega^{k+q} \text { and } \mathrm{d} \mathcal{I}_{\mathscr{X}} \wedge \alpha \subseteq\left(f_{1}, \ldots, f_{k}\right) \Omega^{q+k+1}\right\}
\end{aligned}
$$

where $\left[\begin{array}{c}\alpha \\ f_{1}, \ldots, f_{k}\end{array}\right]$ denotes residue symbols (see [Ker83]). The isomorphism between $\mathcal{R}_{\mathscr{X}}^{q}$ and $\omega_{\mathscr{X}}^{q}$ is then given by: $\sigma: \operatorname{res} \mathscr{X} / \mathscr{C}(\omega) \in \mathcal{R}_{\mathscr{X}}^{q} \mapsto\left[\begin{array}{c}h \omega \\ h_{1}, \ldots, h_{k}\end{array}\right] \in \omega_{\mathscr{X}}^{q}$. Due to $[\operatorname{Ker} 83, \S 2]$, for $\left(f_{1}^{\prime}, \ldots, f_{k}^{\prime}\right) \subseteq$ $\left\langle f_{1}, \ldots, f_{k}\right\rangle$ a regular sequence, we have $\left[\begin{array}{c}\alpha \\ f_{1}, \ldots, f_{k}\end{array}\right]=\left[\begin{array}{c}\Delta \alpha \\ f_{1}^{\prime}, \ldots, f_{k}^{\prime}\end{array}\right]$ where $\Delta$ is the determinant of the transition matrix from $\left(f_{1}, \ldots, f_{k}\right)$ to $\left(f_{1}^{\prime}, \ldots, f_{k}^{\prime}\right)$. If $\left(f_{1}, \ldots, f_{k}\right)$ is an homogeneous regular sequence, it is then convenient to set $\operatorname{deg}\left(\left[\begin{array}{c}\alpha \\ f_{1}, \ldots, f_{k}\end{array}\right]\right)=\operatorname{deg}(\alpha)-\operatorname{deg}\left(f_{1} \cdots f_{k}\right)$ so that the degree does not depend on the choice of the homogeneous regular sequence $\left(f_{1}, \ldots, f_{k}\right)$. It is then easy to see that $\sigma$ has degree $k$.

2.2.2. Characteristic polynomial. We recall here some useful combinatorial invariants associated with a subspace arrangement which can be found for example in [Bjo94].

We first recall the definition of the Hilbert-Poincare series associated with a finitely generated graded $S$-module:

Definition 2.24. Let $M=\bigoplus_{p \geqslant p_{0}}$ be a finitely generated graded $S$-module such that each $M_{p}$ is finite dimensional over $\mathbb{C}$. The Hilbert-Poincaré series of $M$ is:

$$
\operatorname{Poin}(M, x)=\sum_{p \geqslant p_{0}}\left(\operatorname{dim}_{\mathbb{C}} M_{p}\right) x^{p} .
$$

The following definition generalizes the Solomon-Poincaré polynomial defined in [OT92, Defintion 4.130]:

Definition 2.25. Let $\left(M^{q}\right)_{0 \leqslant q \leqslant n}$ be a finite sequence of finitely generated graded $S$-modules. We define the $\Psi$-function associated with $\left(M^{q}\right)_{q}$ as:

$$
\Psi\left(M^{\bullet}, x, t\right)=\sum_{q=0}^{n} \operatorname{Poin}\left(M^{q}, x\right)(t(1-x)-1)^{q} .
$$

Notation 2.26. The intersection lattice $L(\mathscr{X})$ of $\mathscr{X}$ is defined by:

$$
L(\mathscr{X})=\left\{\bigcap_{i \in I} \mathscr{X}_{i} ; I \subseteq\{1, \ldots, s\}\right\} .
$$

In particular, if $I=\emptyset$, we set $\bigcap_{i \in I} \mathscr{X}_{i}=\mathbb{C}^{\ell}$. We consider the partial order on $L(\mathscr{X})$ given by reverse inclusion, so that for all $X, Y \in L(\mathscr{X}), X \leqslant Y$ means $Y \subseteq X$.

Definition 2.27. The Möbius function is defined on $L(\mathscr{X}) \times L(\mathscr{X})$ by:

(1) for all $X \in L(\mathscr{X}), \mu(X, X)=1$,

(2) for all $X, Y \in L(\mathscr{X})$ such that $X<Y, \sum_{\substack{Z \in L(\mathscr{X}) \\ X \leqslant Z \leqslant Y}} \mu(X, Z)=0$,

(3) $\mu(X, Y)=0$ otherwise.

For all $X \in L(\mathscr{X})$, we set $\mu(X)=\mu(V, X)$. 
Definition 2.28 ([Bjo94, (4.4.1)]). The characteristic polynomial of $\mathscr{X}$ is defined by:

$$
\chi(\mathscr{X}, t)=\sum_{X \in L(\mathscr{X})} \mu(X) t^{\operatorname{dim}(X)} .
$$

The purpose of this paper is to study a generalization of the following theorem:

Theorem 2.29 (Solomon Terao formula, [OT92, Theorem 4.136]). Let $\mathscr{A}$ be an hyperplane arrangement in $\mathbb{C}^{\ell}$. Then:

$$
\chi(\mathscr{A}, t)=\Psi\left(\Omega^{\bullet}(\log \mathscr{A}), 1, t\right) .
$$

\section{Properties of the $\Psi$-FunCtion}

We investigate some properties of the $\Psi$-functions associated with the modules of multi-logarithmic forms $\Omega^{\bullet}(\log \mathscr{X} / \mathscr{C})$, the modules $\frac{1}{h} \mathcal{I}_{\mathscr{C}} \Omega^{\bullet}$ and the multi-residues $\mathcal{R}_{\mathscr{X}}^{\bullet}$ for an equidimensional subspace arrangement $\mathscr{X}$ contained in a reduced complete intersection subspace arrangement $\mathscr{C}$.

3.1. Polynomial. Let us prove the following property, which generalizes [OT92, Proposition 4.133].

Proposition 3.1. The $\Psi$-function associated with the modules of multi-logarithmic forms $\Omega^{\bullet}(\log \mathscr{X} / \mathscr{C})$ satisfies:

$$
\Psi\left(\Omega^{\bullet}(\log \mathscr{X} / \mathscr{C}), x, t\right) \in \mathbb{Z}\left[x, x^{-1}, t\right] .
$$

Proof. The proof we suggest here is a generalization of the proof of [OT92, Proposition 4.133] in the context of subspace arrangements. Given a vector space $W$ we will denote by $\Omega^{q}[W]$ the module of $q$-differential forms on $W$. For $p \in \mathbb{N}$, we denote by $\Omega^{q}[W]_{p}$ the module of homogeneous $q$-forms of degree $p$ on $W$. We set $V=\mathbb{C}^{\ell}$. In particular, we have $\Omega^{q}=\Omega^{q}[V]$.

Lemma 3.2. Let $\eta \in \Omega^{1}$. Then for all $\omega \in \Omega^{q}(\log \mathscr{X} / \mathscr{C}), \eta \wedge \omega \in \Omega^{q+1}(\log \mathscr{X} / \mathscr{C})$.

Proof. It comes from the fact that $\eta \wedge \frac{1}{h} \mathcal{I}_{\mathscr{C}} \Omega^{q} \subseteq \frac{1}{h} \mathcal{I}_{\mathscr{C}} \Omega^{q+1}$.

Definition 3.3. Let $\eta \in \Omega^{1}$ be an homogeneous 1 -form of degree $p$. For all $q \in\{0, \ldots, \ell-1\}$ we define $\partial_{\eta}: \Omega^{q}(\log \mathscr{X} / \mathscr{C}) \rightarrow \Omega^{q+1}(\log \mathscr{X} / \mathscr{C})$ by $\partial_{\eta}(\omega)=\eta \wedge \omega$. This map is homogeneous of degree $p$. The $\eta$-complex is the complex:

$$
0 \rightarrow \Omega^{0}(\log \mathscr{X} / \mathscr{C}) \stackrel{\partial_{\eta}}{\longrightarrow} \Omega^{1}(\log \mathscr{X} / \mathscr{C}) \stackrel{\partial_{\eta}}{\longrightarrow} \ldots \stackrel{\partial_{\eta}}{\longrightarrow} \Omega^{\ell}(\log \mathscr{X} / \mathscr{C}) \rightarrow 0 .
$$

Our purpose is to prove that for a generic $\eta$, the cohomology groups of the $\eta$-complex are finite dimensional over $\mathbb{C}$ as in [OT92, Proposition 4.91].

We consider the Zariski topology on the vector subspace $S_{p}$ of $S$ composed of the homogeneous polynomials of degree $p$.

We recall that by [OT92, Lemma 4.88], if $W$ is an $m$-dimensional $\mathbb{C}$-vector space, a generic $\omega \in \Omega^{1}[W]_{p}$ vanishes only at the origin.

Let $X \in L(\mathscr{X})$ with $\operatorname{dim}(X)>0$. As in [OT92, Lemma 4.89], the restriction map

$$
r_{V, X}: \Omega_{p}^{1} \rightarrow \Omega^{1}[X]_{p}
$$

is continuous with respect to the Zariski topology. We set

$$
N_{p}^{X}=\left\{\omega \in \Omega_{p}^{1} ; r_{V, X}(\omega) \text { vanishes only at the origin of } X\right\} .
$$

The set $N_{p}^{X}$ is an open dense subset in $\Omega_{p}^{1}$ since $r_{V, X}$ is continuous.

Notation 3.4. We set:

$$
N_{p}=\bigcap_{\substack{X \in L(\mathscr{X}) \\ \operatorname{dim}(X)>0}} N_{p}^{X}
$$

Since $L(\mathscr{X})$ is finite, $N_{p}$ is an open dense subset of $\Omega_{p}^{1}$.

Definition 3.5. The module of logarithmic vector fields along $\mathscr{X}$ is defined by

$$
\operatorname{Der}(-\log \mathscr{X})=\left\{\delta \in \Theta ; \delta\left(\mathcal{I}_{\mathscr{X}}\right) \subseteq \mathcal{I}_{\mathscr{X}}\right\} .
$$


Lemma 3.6. Let $\eta \in N_{p}$. Then the radical of the ideal

$$
I(\eta)=\{\langle\eta, \delta\rangle, \delta \in \operatorname{Der}(-\log \mathscr{X})\}
$$

contains the maximal ideal $S_{+}=\bigoplus_{q>0} S_{q}$.

Proof. It suffices to prove that the zero locus of $I(\eta)$ satisfies $V(I(\eta)) \subseteq\{0\}$. Let $v \in S \backslash\{0\}$.

If $v \notin \bigcup_{i=1}^{s} \mathscr{X}_{i}$, let $Q \in \mathcal{I}_{\mathscr{X}}$ be such that $Q(v) \neq 0$. Since $N_{p} \subseteq N_{p}^{V}$, if we write $\eta=\sum_{i=1}^{\ell} \alpha_{i} \mathrm{~d} x_{i}$, there exists $i \in\{1, \ldots, \ell\}$ such that $\alpha_{i}(v) \neq 0$. Then $Q \frac{\partial}{\partial_{x_{i}}} \in \operatorname{Der}(-\log \mathscr{X})$ and $\left\langle Q \frac{\partial}{\partial x_{i}}, \eta\right\rangle=Q \alpha_{i}$ does not vanish on $v$, so that $v \notin V(I(\eta))$.

Let us assume that $v \in \bigcup_{i=1}^{s} \mathscr{X}_{i}$. Let $X=\bigcap_{\mathscr{X}_{i} \ni v} \mathscr{X}_{i}$. We choose a basis $\left(y_{1}, \ldots, y_{\ell}\right)$ of $V^{*}$ such that $X$ is defined by $y_{m+1}=\cdots=y_{\ell}=0$. Let

$$
J_{1}=\left\{i \in\{1, \ldots, s\} ; X \nsubseteq \mathscr{X}_{i}\right\} \text { and } J_{2}=\left\{i \in\{1, \ldots, s\} ; X \subseteq \mathscr{X}_{i}\right\}=\{1, \ldots, s\} \backslash J_{1} .
$$

Let us denote $\mathscr{Z}_{1}=\bigcup_{i \in J_{1}} \mathscr{X}_{i}$ and $\mathscr{Z}_{2}=\bigcup_{i \in J_{2}} \mathscr{X}_{i}$. In particular, $v \notin \mathscr{Z}_{1}$. Let $Q \in \mathcal{I}_{\mathscr{Z}_{1}}$ be such that $Q(v) \neq 0$. Let us prove that for all $i \in\{1, \ldots, m\}$, we have $Q \frac{\partial}{\partial y_{i}} \in \operatorname{Der}(-\log \mathscr{X})$.

Let $i \in\{1, \ldots, m\}$. We have to prove that for all $h \in \mathcal{I}_{\mathscr{X}}, Q \frac{\partial}{\partial y_{i}}(h) \in \mathcal{I}_{\mathscr{X}}$. Since $\mathcal{I}_{\mathscr{X}}$ is radical, it suffices to prove that for all $j \in\{1, \ldots, s\}$, the restriction of $Q \frac{\partial}{\partial y_{i}}(h)$ to $\mathscr{X}_{j}$ is zero. It is clear that for all $j \in J_{1},\left.Q \frac{\partial}{\partial_{y_{i}}}(h)\right|_{\mathscr{X}_{j}}=0$ since $Q \in \mathcal{I}\left(\mathscr{Z}_{1}\right)$. Let $j \in J_{2}$. A generating family $\left(g_{1}, \ldots, g_{k}\right)$ of $\mathcal{I}_{\mathscr{X}_{j}}$ can be chosen so that for all $n \in\{1, \ldots, k\}, g_{n}$ is homogeneous of degree 1 . Since $X \subseteq \mathscr{X}_{j} \subseteq \mathscr{X}$, we have $\mathcal{I}_{\mathscr{X}} \subseteq \mathcal{I}_{\mathscr{X}_{j}} \subseteq \mathcal{I}_{X}=\left\langle y_{m+1}, \ldots, y_{\ell}\right\rangle_{S}$. Therefore, for all $n \in\{1, \ldots, k\}, g_{n} \in\left\langle y_{m+1}, \ldots, y_{\ell}\right\rangle_{\mathbb{C}}$ so that for all $n \in\{1, \ldots, k\}, Q \frac{\partial}{\partial_{y_{i}}}\left(g_{n}\right)=0$. Thus, for all $h=\sum a_{n} g_{n} \in \mathcal{I}_{\mathscr{X}},\left.Q \frac{\partial}{\partial_{y_{i}}}(h)\right|_{\mathscr{X}_{j}}=$ $\left.\left(\sum g_{n} \frac{\partial a_{n}}{\partial y_{i}}\right)\right|_{\mathscr{X}_{j}}=0$. Hence the result: $Q \frac{\partial}{\partial_{y_{i}}} \in \operatorname{Der}(-\log \mathscr{X})$.

There exist $\left(\alpha_{1}, \ldots, \alpha_{\ell}\right) \in\left(S_{p}\right)^{\ell}$ such that $\eta=\sum_{n=1}^{\ell} \alpha_{n} \mathrm{~d} y_{n}$. Then for all $i \in\{1, \ldots, m\}$, $\left\langle Q \frac{\partial}{\partial y_{i}}, \eta\right\rangle=Q \alpha_{i}$. The restriction of $\eta$ to $X$ is:

$$
r_{S, X}(\eta)=\overline{\alpha_{1}} \mathrm{~d} \overline{y_{1}}+\cdots+\overline{\alpha_{m}} \mathrm{~d} \overline{y_{m}} .
$$

Since $\eta \in N_{p}^{X}$, it vanishes only at the origin. Therefore, there exists $i \in\{1, \ldots, m\}$ such that $\alpha_{i}(v)=\overline{\alpha_{i}}(v) \neq 0$. Since $Q(v) \neq 0$, we have $v \notin V(I(\eta))$.

Lemma 3.7. Let $\omega \in \Omega^{q}(\log \mathscr{X} / \mathscr{C})$ and $\theta \in \operatorname{Der}(-\log \mathscr{X})$. Then $\langle\omega, \theta\rangle \in \Omega^{q-1}(\log \mathscr{X} / \mathscr{C})$.

Proof. By theorem 2.10, we can write:

$$
g \omega=\frac{c_{\mathscr{X}} / \mathscr{C}}{h} \wedge \xi+\lambda
$$

with $\lambda \in \frac{1}{h} \mathcal{I}_{\mathscr{C}} \Omega^{q}$. Then:

$$
\begin{aligned}
g\langle\omega, \theta\rangle & =\langle g \omega, \theta\rangle=\left\langle\frac{c_{\mathscr{X} / \mathscr{C}}}{h} \wedge \xi, \theta\right\rangle+\langle\lambda, \theta\rangle \\
& =\left\langle\frac{c_{\mathscr{X} / \mathscr{C}}}{h}, \theta\right\rangle \wedge \xi+(-1)^{k} \frac{c_{\mathscr{X} / \mathscr{C}}}{h} \wedge\langle\xi, \theta\rangle+\langle\lambda, \theta\rangle
\end{aligned}
$$

Let us prove that $\left\langle c_{\mathscr{X} / \mathscr{C}}, \theta\right\rangle \in \mathcal{I}_{\mathscr{C}} \Omega^{k-1}$. It suffices to prove that $\overline{\left\langle c_{\mathscr{X} / \mathscr{C}}, \theta\right\rangle} \in \Omega^{k-1} \otimes \operatorname{Frac}\left(\mathcal{O}_{\mathscr{C}}\right)$ is zero. We have:

$$
\begin{aligned}
\overline{\left\langle c_{\mathscr{X} / \mathscr{C}}, \theta\right\rangle} & =\overline{\left\langle\beta_{\mathscr{X} / \mathscr{C}} \mathrm{d} h_{1} \wedge \cdots \wedge \mathrm{d} h_{k}, \theta\right\rangle} \\
& =\overline{\sum_{i=1}^{k}(-1)^{i-1} \beta_{\mathscr{X} / \mathscr{C}}\left\langle\mathrm{d} h_{i}, \theta\right\rangle \mathrm{d} h_{1} \wedge \cdots \wedge \widehat{\mathrm{dh}}_{i} \wedge \cdots \wedge \mathrm{d} h_{k}}
\end{aligned}
$$


Since $\theta \in \operatorname{Der}(-\log \mathscr{X}),\left\langle\mathrm{d} h_{i}, \theta\right\rangle \in \mathcal{I}_{\mathscr{X}}$. In addition, we deduce from the definition of $\beta_{\mathscr{X} / \mathscr{C}}$ that $\left.\beta_{\mathscr{X} / \mathscr{C}}\left\langle\mathrm{d} h_{i}, \theta\right\rangle\right|_{\mathscr{C}}=0$, since $\beta_{\mathscr{X} / \mathscr{C}}$ is zero on the components of $\mathscr{C}$ which are not in $\mathscr{X}$.

Therefore, the form $\langle\omega, \theta\rangle$ satisfies theorem 2.10 and $\langle\omega, \theta\rangle \in \Omega^{q-1}(\log \mathscr{X} / \mathscr{C})$.

Remark 3.8. The previous lemma is not specific to subspace arrangements. Let $X \subseteq\left(\mathbb{C}^{\ell}, 0\right)$ be a reduced equidimensional subspace and $C$ a reduced complete intersection of the same dimension as $X$. Let $\operatorname{Der}(-\log X)=\left\{\delta \in \Theta ; \delta\left(\mathcal{I}_{X}\right) \subseteq \mathcal{I}_{X}\right\}$. With the same proof as for lemma 3.7, we obtain that for all $\omega \in \Omega^{q}(\log X / C)$ and $\theta \in \operatorname{Der}(-\log X),\langle\omega, \theta\rangle \in \Omega^{q-1}(\log X / C)$.

Thanks to lemmas 3.6 and 3.7, [OT92, Proposition 4.91] can be generalized to subspace arrangements with a completely similar proof and gives:

Proposition 3.9. If $\eta \in N_{p}$, then the cohomology groups of the $\eta$-complex are finite dimensional over $\mathbb{C}$.

By considering minimal integers $m, n \in \mathbb{N}$ such that $P(x, t)=x^{n}(1-x)^{m} \Psi\left(\Omega^{\bullet}(\log \mathscr{X} / \mathscr{C}), x, t\right)$ is a polynomial in $x$ and $t$ and using proposition 3.9, one can prove in a similar way as for [OT92, Proposition 4.133] that $m=0$, so that $\Psi\left(\Omega^{\bullet}(\log \mathscr{X} / \mathscr{C}), x, t\right)$ is a polynomial in $x, x^{-1}$ and $t$.

3.2. Decomposition. The purpose of this subsection is to distinguish in the $\Psi$-function of $\Omega^{\bullet}(\log \mathscr{X} / \mathscr{C})$ the contribution of the modules of multi-residues, which are intrinsic, from the contribution of the modules $\frac{1}{h} \mathcal{I}_{\mathscr{C}} \Omega^{\bullet}$ which depend on the choice of the complete intersection $\mathscr{C}$.

Since $\mathcal{I}_{\mathscr{C}}$ is generated by a regular sequence, a free resolution of the modules $\frac{1}{h} \mathcal{I}_{\mathscr{C}} \Omega^{q}$ is deduced from the Koszul complex for all $q$, so that we can compute explicitly $\Psi\left(\frac{1}{h} \mathcal{I}_{\mathscr{C}} \Omega^{\bullet}, x, t\right)$.

Proposition 3.10. We have:

$$
\Psi\left(\frac{1}{h} \mathcal{I}_{\mathscr{C}} \Omega^{\bullet}, x, t\right)=t^{\ell} x^{-d}\left(1-\prod_{i=1}^{k}\left(1-x^{d_{i}}\right)\right) .
$$

Proof. Since for all $q \in \mathbb{N}$ the module $\Omega^{q}$ is a free $S$-module of rank $\left(\begin{array}{l}\ell \\ q\end{array}\right)$, we have:

$$
\operatorname{Poin}\left(\frac{1}{h} \mathcal{I}_{\mathscr{C}} \Omega^{q}, x\right)=\left(\begin{array}{l}
\ell \\
q
\end{array}\right) \operatorname{Poin}\left(\frac{1}{h} \mathcal{I}_{\mathscr{C}}, x\right)
$$

A free resolution of $\frac{1}{h} \mathcal{I}_{\mathscr{C}}$ is deduced from the Koszul complex associated with the regular sequence $\left(h_{1}, \ldots, h_{k}\right)$. We recall that for all $i \in\{1, \ldots, k\}, \operatorname{deg}\left(h_{i}\right)=d_{i}$ and $d=\operatorname{deg}(h)$. We have:

$$
0 \rightarrow S(0) \rightarrow \cdots \rightarrow \bigoplus_{1 \leqslant i_{1}<i_{2} \leqslant k} S\left(-d_{i_{1}}-d_{i_{2}}+d\right) \rightarrow \bigoplus_{1 \leqslant i_{1} \leqslant k} S\left(-d_{i_{1}}+d\right) \rightarrow \frac{1}{h} \mathcal{I}_{\mathscr{C}} \rightarrow 0 .
$$

Therefore:

$$
\begin{aligned}
\operatorname{Poin}\left(\frac{1}{h} \mathcal{I}_{\mathscr{C}}, x\right) & =\sum_{j=1}^{k}(-1)^{j-1} \sum_{1 \leqslant i_{1}<\ldots<i_{j} \leqslant k} x^{-d} \frac{x^{d_{i_{1}}+\cdots+d_{i_{j}}}}{(1-x)^{\ell}} \\
& =\frac{1}{x^{d}(1-x)^{\ell}}-\frac{1}{x^{d}(1-x)^{\ell}} \sum_{j=0}^{k} \sum_{1 \leqslant i_{1}<\ldots<i_{j} \leqslant k}(-1)^{j} x^{d_{i_{1}}+\cdots+d_{i_{j}}} \\
& =\frac{1}{x^{d}(1-x)^{\ell}}-\frac{\left(1-x^{d_{1}}\right) \ldots\left(1-x^{d_{k}}\right)}{x^{d}(1-x)^{\ell}}
\end{aligned}
$$


Thus:

$$
\begin{aligned}
\Psi\left(\frac{1}{h} \mathcal{I}_{\mathscr{C}} \Omega^{\bullet}, x, t\right) & =\sum_{q=0}^{\ell}\left(\begin{array}{l}
\ell \\
q
\end{array}\right) \operatorname{Poin}\left(\frac{1}{h} \mathcal{I}_{\mathscr{C}}, x\right)(t(1-x)-1)^{q} \\
& =\operatorname{Poin}\left(\frac{1}{h} \mathcal{I}_{\mathscr{C}}, x\right) \sum_{q=0}^{\ell}\left(\begin{array}{l}
\ell \\
q
\end{array}\right)(t(1-x)-1)^{q} \\
& =\left(\frac{1-\left(1-x^{d_{1}}\right) \ldots\left(1-x^{d_{k}}\right)}{x^{d}(1-x)^{\ell}}\right)(t(1-x))^{\ell} \\
& =t^{\ell}\left(\frac{1-\left(1-x^{d_{1}}\right) \ldots\left(1-x^{d_{k}}\right)}{x^{d}}\right)
\end{aligned}
$$

Hence the result.

Proposition 3.11. The $\Psi$-functions of $\Omega^{\bullet}(\log \mathscr{X} / \mathscr{C}), \mathcal{R}_{\mathscr{X}}^{\bullet}$ and $\frac{1}{h} \mathcal{I}_{\mathscr{C}} \Omega^{\bullet}$ are related as follows:

$$
\Psi\left(\Omega^{\bullet}(\log \mathscr{X} / \mathscr{C}), x, t\right)=\Psi\left(\frac{1}{h} \mathcal{I}_{\mathscr{C}} \Omega^{\bullet}, x, t\right)+(t(1-x)-1)^{k} x^{-k} \Psi\left(\mathcal{R}_{\mathscr{X}}^{\bullet}, x, t\right)
$$

Proof. We recall the exact sequence (4) which holds for all $q \in \mathbb{N}$ :

$$
0 \rightarrow \frac{1}{h} \mathcal{I}_{\mathscr{C}} \Omega^{q} \rightarrow \Omega^{q}(\log \mathscr{X} / \mathscr{C}) \stackrel{\text { res } \mathscr{X} / \mathscr{C}}{\longrightarrow} \mathcal{R}_{\mathscr{X}}^{q-k} \rightarrow 0 .
$$

By proposition 2.22, the map res $\mathscr{X} / \mathscr{C}$ is homogeneous of degree $k$. Since Hilbert-Poincaré series are additive, we have for all $q \in \mathbb{N}$ :

$$
\operatorname{Poin}\left(\Omega^{q}(\log \mathscr{X} / \mathscr{C}), x\right)=\operatorname{Poin}\left(\frac{1}{h} \mathcal{I}_{\mathscr{C}} \Omega^{q}, x\right)+x^{-k} \operatorname{Poin}\left(\mathcal{R}_{\mathscr{X}}^{q-k}, x\right)
$$

Therefore:

$$
\begin{aligned}
\Psi\left(\Omega^{\bullet}(\log \mathscr{X} / \mathscr{C}), x, t\right) & =\sum_{q=0}^{\ell} \operatorname{Poin}\left(\Omega^{q}(\log \mathscr{X} / \mathscr{C}), x\right)(t(1-x)-1)^{q} \\
& =\sum_{q=0}^{\ell} \operatorname{Poin}\left(\frac{1}{h} \mathcal{I}_{\mathscr{C}} \Omega^{q}, x\right)(t(1-x)-1)^{q}+\sum_{q=k}^{\ell} x^{-k} \operatorname{Poin}\left(\mathcal{R}_{\mathscr{X}}^{q-k}, x\right)(t(1-x)-1)^{q} \\
& =\Psi\left(\frac{1}{h} \mathcal{I}_{\mathscr{C}} \Omega^{\bullet}, x, t\right)+x^{-k}(t(1-x)-1)^{k} \Psi\left(\mathcal{R}_{\mathscr{X}}^{\bullet}, x, t\right)
\end{aligned}
$$

Hence the result.

Remark 3.12. If $\left(h_{1}^{\prime}, \ldots, h_{k}^{\prime}\right) \subseteq \mathcal{I}_{\mathscr{X}}$ is another homogeneous regular sequence defining a reduced complete intersection $\mathscr{C}^{\prime}$, then the two functions $\Psi\left(\Omega^{\bullet}(\log \mathscr{X} / \mathscr{C}), x, t\right)$ and $\Psi\left(\Omega^{\bullet}\left(\log \mathscr{X} / \mathscr{C}^{\prime}\right), x, t\right)$ may be different.

Indeed, if the degrees of the equations of $\mathscr{C}^{\prime}$ are different from the degrees of the equations of $\mathscr{C}$, then by proposition $3.1, \Psi\left(\frac{1}{h} \mathcal{I}_{\mathscr{C}} \Omega^{\bullet}, x, t\right)$ may be different from $\Psi\left(\frac{1}{h^{\prime}} \mathcal{I}_{\mathscr{C}} \Omega^{\bullet}, x, t\right)$. On the contrary, the function $\Psi\left(\mathcal{R}_{\mathscr{X}}^{\bullet}, x, t\right)$ does not depend on the choice of the complete intersection $\mathscr{C}$.

3.3. An explicit computation: the case of complete intersection line arrangements. Let us give an example which is the motivation of the next part of this paper. The computation of the modules of multi-logarithmic forms along a reduced equidimensional subspace is difficult in general. The case of quasi-homogeneous complete intersection curves is explicitly computed in [Pol16b, Théorème 6.1.29 and Théorème 6.1.33].

Proposition 3.13. Let $\mathscr{C} \subseteq \mathbb{C}^{\ell}$ be a reduced homogeneous ${ }^{2}$ complete intersection curve with embedding dimension $\ell$. We suppose that $\mathcal{I}_{\mathscr{C}}$ is generated by a homogeneous regular sequence $\left(h_{1}, \ldots, h_{\ell-1}\right)$.

\footnotetext{
${ }^{2}$ It is not necessary to assume that $\mathscr{C}$ is a line arrangement.
} 
We set $h=h_{1} \cdots h_{\ell-1}$. For all $i \in\{1, \ldots, \ell-1\}$, we denote by $d_{i}$ the degree of $h_{i}$, and $d=\operatorname{deg}(h)$. Then:

$$
\begin{aligned}
\Psi\left(\Omega^{\bullet}(\log \mathscr{C}), x, t\right)= & t^{\ell} x^{-d}\left(1-\prod_{i=1}^{\ell-1}\left(1-x^{d_{i}}\right)\right) \\
& +(t(1-x)-1)^{\ell-1} x^{-\ell+1}\left(1+(t-1) x^{\ell-d-1} \prod_{i=1}^{\ell-1}\left(1+x+\cdots+x^{d_{i}-1}\right)\right) .
\end{aligned}
$$

In particular,

$$
\Psi\left(\Omega^{\bullet}(\log \mathcal{C}), 1, t\right)=t^{\ell}+(-1)^{\ell-1}\left(d_{1} \cdots d_{\ell-1}\right) t+(-1)^{\ell}\left(d_{1} \cdots d_{\ell-1}-1\right) .
$$

In particular, if $\mathscr{C}$ is a line arrangement, then $\mathscr{C}$ has $d_{1} \cdots d_{\ell-1}$ components and we obtain the following property which generalizes Solomon-Terao formula 2.29:

Corollary 3.14. If in addition the reduced complete intersection $\mathscr{C}$ is a line arrangement, the characteristic polynomial of $\mathscr{C}$ is:

$$
\chi(\mathscr{C}, t)=t^{\ell}-d_{1} \cdots d_{\ell-1} t+\left(d_{1} \cdots d_{\ell-1}-1\right) .
$$

We thus have:

$$
\chi(\mathscr{C}, t)=t^{\ell}-\Psi\left(\mathcal{R}_{\mathscr{C}}^{\bullet}, 1, t\right) .
$$

In particular, if $\ell-1$ is odd,

$$
\Psi\left(\Omega^{\bullet}(\log \mathscr{C}), 1, t\right)=\chi(\mathscr{C}, t) .
$$

Proof of proposition 3.13. Thanks to proposition 3.11 and 3.10, it is sufficient to compute the $\Psi$ function relative to the module of logarithmic multi-residues. Let us compute $\operatorname{Poin}\left(\mathcal{R}_{\mathscr{C}}, x\right)$ and $\operatorname{Poin}\left(\mathcal{R}_{\mathscr{C}}^{1}, x\right)$.

By [Pol16b, Théorème 6.1.29], a free resolution of $\mathcal{R}_{\mathscr{C}}$ is given by:

$$
\begin{aligned}
0 \rightarrow S(-(\ell-1))^{\left(\begin{array}{c}
\ell \\
\ell-1
\end{array}\right)} & \rightarrow \bigoplus_{1 \leqslant i_{1}<\ldots<i_{\ell-2} \leqslant \ell-1} S\left(d-\ell-d_{i_{1}}-\cdots-d_{i_{\ell-2}}\right) \oplus S(-(\ell-2))^{\left(\begin{array}{c}
\ell \\
\ell-2
\end{array}\right)} \rightarrow \ldots \\
\cdots & \rightarrow \bigoplus_{1 \leqslant i_{1} \leqslant \ell-1} S\left(d-\ell-d_{i_{1}}\right) \oplus S(-1)^{\left(\begin{array}{l}
\ell \\
1
\end{array}\right)} \rightarrow S(d-\ell) \oplus S(0) \rightarrow \mathcal{R}_{\mathscr{C}} \rightarrow 0 .
\end{aligned}
$$

We then have:

$$
\begin{aligned}
\operatorname{Poin}\left(\mathcal{R}_{\mathscr{C}}, x\right)= & \sum_{j=0}^{\ell-1}(-1)^{j}\left(\begin{array}{l}
\ell \\
j
\end{array}\right) \frac{x^{j}}{(1-x)^{\ell}}+\sum_{j=0}^{\ell-2}(-1)^{j} x^{\ell-d} \sum_{1 \leqslant i_{1}<\ldots<i_{j} \leqslant \ell-1} \frac{x^{d_{i_{1}}+\cdots+d_{i_{j}}}}{(1-x)^{\ell}} \\
= & (-1)^{\ell-1} \frac{x^{\ell}}{(1-x)^{\ell}}+\frac{1}{(1-x)^{\ell}} \sum_{j=0}^{\ell}\left(\begin{array}{c}
\ell \\
j
\end{array}\right)(-x)^{j} \\
& +(-1)^{\ell} x^{\ell-d} \frac{x^{d}}{(1-x)^{\ell}}+\frac{x^{\ell-d}}{(1-x)^{\ell}} \sum_{j=0}^{\ell-1} \sum_{1 \leqslant i_{1}<\ldots<i_{j} \leqslant \ell-1}(-1)^{j}\left(x^{d_{i_{1}}}+\cdots+x^{d_{i_{j}}}\right) \\
= & 1+\frac{x^{\ell-d}}{(1-x)^{\ell}}\left(1-x^{d_{1}}\right) \cdots\left(1-x^{d_{\ell-1}}\right) .
\end{aligned}
$$

Let us compute $\operatorname{Poin}\left(\mathcal{R}_{\mathscr{C}}^{1}, x\right)$. We recall that $\mathcal{R}_{\mathscr{C}}^{1}=\operatorname{res}_{\mathscr{C}}\left(\Omega^{\ell}(\log \mathscr{C})\right)$. In addition, it is easy to see that $\Omega^{\ell}(\log \mathscr{C})=\frac{1}{h} \Omega^{m}$ is a free $S$-module of rank 1 . Therefore, $\mathcal{R}_{\mathscr{C}}^{1}$ is the free $\mathcal{O}_{\mathscr{C}}$-module generated by $\operatorname{res}_{\mathscr{C}}\left(\frac{\mathrm{d} x_{1} \wedge \cdots \wedge \mathrm{d} x_{\ell}}{h}\right)$. A free resolution of $\mathcal{R}_{\mathscr{C}}^{1}$ can therefore be deduced from the Koszul complex associated with $\left(h_{1}, \ldots, h_{\ell-1}\right)$ : 
(7)

$$
\begin{aligned}
0 \rightarrow S(-\ell+1) \rightarrow \bigoplus_{1 \leqslant i_{1}<\ldots<i_{\ell-2} \leqslant \ell-1} S\left(d-\ell+1-d_{i_{1}}-\cdots-d_{i_{\ell-2}}\right) & \rightarrow \ldots \\
\cdots & \rightarrow \bigoplus_{1 \leqslant i_{1} \leqslant \ell-1} S\left(d-\ell+1-d_{i_{1}}\right) \rightarrow S(d-\ell+1) \rightarrow \mathcal{R}_{\mathscr{C}}^{1} \rightarrow 0 .
\end{aligned}
$$

Therefore, we have:

$$
\begin{aligned}
\operatorname{Poin}\left(\mathcal{R}_{\mathscr{C}}^{1}, x\right) & =\sum_{j=0}^{\ell-1}(-1)^{j} \sum_{1 \leqslant i_{1}<\ldots<i_{j} \leqslant \ell-1} x^{\ell-d-1} \frac{x^{d_{i_{1}}}+\cdots+x^{d_{i_{j}}}}{(1-x)^{\ell}} \\
& =\frac{x^{\ell-d-1}}{(1-x)^{\ell}}\left(1-x^{d_{1}}\right) \cdots\left(1-x^{d_{\ell-1}}\right)
\end{aligned}
$$

We then compute the $\Psi$-function associated with the modules of logarithmic multi-residues:

$$
\begin{aligned}
\Psi\left(\mathcal{R}_{\mathscr{C}}^{\bullet}, x, t\right) & =\operatorname{Poin}\left(\mathcal{R}_{\mathscr{C}}, x\right)+(t(1-x)-1) \operatorname{Poin}\left(\mathcal{R}_{\mathscr{C}}^{1}, x\right) \\
& =1+\frac{x^{\ell-d}}{(1-x)^{\ell}}\left(1-x^{d_{1}}\right) \cdots\left(1-x^{d_{\ell-1}}\right)+(t(1-x)-1) \frac{x^{\ell-d-1}}{(1-x)^{\ell}}\left(1-x^{d_{1}}\right) \cdots\left(1-x^{d_{\ell-1}}\right) \\
& =1+\frac{x^{\ell-d-1}}{(1-x)^{\ell}}\left(1-x^{d_{1}}\right) \cdots\left(1-x^{d_{\ell-1}}\right)(x+t(1-x)-1) \\
& =1+\frac{x^{\ell-d-1}}{(1-x)^{\ell}}\left(1-x^{d_{1}}\right) \cdots\left(1-x^{d_{\ell-1}}\right)(1-x)(t-1) \\
& =1+x^{\ell-d-1}(t-1) \prod_{i=1}^{\ell-1}\left(1+x+\cdots+x^{d_{i}-1}\right)
\end{aligned}
$$

This computation and propositions 3.10 and 3.11 show that $\Psi\left(\Omega^{\bullet}(\log \mathscr{C}), x, t\right)$ is given by (5).

\section{Solomon-Terao Formula for subspace ARRAngements}

We investigate in this section a generalization of Solomon-Terao formula 2.29 for subspace arrangements, which leads to our main theorem 4.2.

4.1. Statement of the main theorem. We assume that $\mathscr{X}=\left\{\mathscr{X}_{1}, \ldots, \mathscr{X}_{s}\right\}$ is a reduced equidimensional subspace arrangement of codimension $k$ in $\mathbb{C}^{\ell}$, and that $\mathscr{C}$ is a reduced complete intersection subspace arrangement of codimension $k$ which contains $\mathscr{X}$. We denote by $\left(h_{1}, \ldots, h_{k}\right)$ an homogeneous regular sequence defining $\mathscr{C}$, and $h=h_{1} \cdots h_{k}$. We recall that $d_{i}$ denotes the degree of $h_{i}$ for all $i \in\{1, \ldots, k\}$.

As suggested by the case of complete intersection line arrangements, we will distinguish the part of the $\Psi$-function of $\Omega^{\bullet}(\log \mathscr{X} / \mathscr{C})$ which comes from $\mathcal{R}_{\mathscr{X}}^{\bullet}$ from the part which comes from $\frac{1}{h} \mathcal{I}_{\mathscr{C}} \Omega^{\bullet}$. We will use the following notations:

Notation 4.1. Let $Y \in L(\mathscr{X})$. We denote $L_{Y}=\{Z \in L(\mathscr{X}) ; Y \subseteq Z\}$. If $Y \in L(\mathscr{X})$, we set $\mathscr{X}_{Y}=\left\{\mathscr{X}_{i} ; Y \subseteq \mathscr{X}_{i}\right\} \subseteq \mathscr{X}$.

Our main theorem is:

TheOREM 4.2. Let $\mathscr{X}$ be a reduced equidimensional subspace arrangement in $\mathbb{C}^{\ell}$ with codimension $k$. Let $\mathscr{C}$ be a reduced complete intersection subspace arrangement of codimension $k$ containing $\mathscr{X}$. If for all $Y \in L(\mathscr{X}) \backslash\{V\}$ we have $\Psi\left(\mathcal{R}_{\mathscr{X}_{Y}}, 1,1\right)=1$ then for all $Y \in L(\mathscr{X})$, we have:

$$
\begin{aligned}
\chi\left(\mathscr{X}_{Y}, t\right) & =\Psi\left(\frac{1}{h} \mathcal{I}_{\mathscr{C}} \Omega^{\bullet}, 1, t\right)-\Psi\left(\mathcal{R}_{\mathscr{X}_{Y}}^{\bullet}, 1, t\right) \\
& =t^{\ell}-\Psi\left(\mathcal{R}_{\mathscr{X}_{Y}}^{\bullet}, 1, t\right) .
\end{aligned}
$$


In particular, if $k$ is odd,

$$
\chi\left(\mathscr{X}_{Y}, t\right)=\Psi\left(\Omega^{\bullet}\left(\log \mathscr{X}_{Y} / \mathscr{C}\right), 1, t\right) .
$$

Remark 4.3. We will prove in section 5 that the condition $\Psi\left(\mathcal{R}_{\mathscr{X}_{Y}}, 1,1\right)=1$ is always satisfied for any line arrangement of any codimension, but is not necessarily satisfied for subspace arrangements of higher dimension.

Remark 4.4. From remark 2.23, one can notice that equation (8) can be rephrased as

$$
\chi\left(\mathscr{X}_{Y}, t\right)=t^{\ell}-\Psi\left(\omega_{\mathscr{X}_{Y}}^{\bullet}, 1, t\right)
$$

since $\Psi\left(\omega_{\mathscr{X}_{Y}}^{\bullet}, x, t\right)=x^{k} \Psi\left(\mathcal{R}_{\mathscr{X}_{Y}}^{\bullet}, x, t\right)$.

4.2. Proof of theorem 4.2. Let us introduce the following function.

Notation 4.5. For $Y \in L(\mathscr{X})$, we define:

$$
\widetilde{\Psi}\left(\mathscr{X}_{Y}, x, t\right)=\Psi\left(\frac{1}{h} \mathcal{I}_{\mathscr{C}} \Omega^{\bullet}, x, t\right)+(-1)^{k+1}(t(1-x)-1)^{k} x^{-k} \Psi\left(\mathcal{R}_{\mathscr{X}_{Y}}, x, t\right) \in \mathbb{Z}\left[x, x^{-1}, t\right] .
$$

In particular, $\widetilde{\Psi}\left(\mathscr{X}_{Y}, 1, t\right)=\Psi\left(\frac{1}{h} \mathcal{I}_{\mathscr{C}} \Omega^{\bullet}, 1, t\right)-\Psi\left(\mathcal{R}_{\mathscr{X}_{Y}}, 1, t\right)=t^{\ell}-\Psi\left(\mathcal{R}_{\mathscr{X}_{Y}}, 1, t\right)$, and if $k$ is odd, we have $\widetilde{\Psi}\left(\mathscr{X}_{Y}, x, t\right)=\Psi\left(\Omega^{\bullet}\left(\log \mathscr{X}_{Y} / \mathscr{C}\right), x, t\right)$.

The proof of [OT92, Proposition 4.135] can be extended to subspace arrangements, so that we have:

Proposition 4.6 ([OT92, Proposition 4.135]). If $G: L(\mathscr{X}) \rightarrow \mathbb{Z}[t]$ is a map satisfying the following four conditions:

a) $G(V)=t^{\ell}$

b) for $Y \neq V,\left.G(Y)\right|_{t=1}=0$,

c) for all $Y \in L(\mathscr{X}), t^{\operatorname{dim} Y}$ divides $G(Y)$,

d) for all $Y \in L(\mathscr{X}), \operatorname{deg}_{t}\left(\sum_{Z \in L_{Y}} \mu(Z, Y) G(Z)\right) \leqslant \operatorname{dim} Y$,

then for all $Y \in L(\mathscr{X}), G(Y)=\chi\left(\mathscr{X}_{Y}, t\right)$.

Notation 4.7. For $Y \in L(\mathscr{X})$, we set $G(Y)=\widetilde{\Psi}\left(\mathscr{X}_{Y}, 1, t\right) \in \mathbb{Z}[t]$.

To prove theorem 4.2, it suffices to prove that $G$ satisfies the properties of proposition 4.6. Let us study each property.

4.2.1. Property (a). For the empty arrangement, $\mathcal{R}_{\emptyset}^{q}=0$ for all $q \in \mathbb{N}$ so that, using proposition 3.10 we obtain the first property (a):

$$
G(V)=\Psi\left(\frac{1}{h} \mathcal{I}_{\mathscr{C}} \Omega^{\bullet}, 1, t\right)=t^{\ell}
$$

Remark 4.8. One can notice that for $q \in \mathbb{N}, \Omega^{q}(\log \emptyset / \mathscr{C})=\frac{1}{h} \mathcal{I}_{\mathscr{C}} \Omega^{q}$ whereas $\Omega^{q}(\log \emptyset)=\Omega^{q}$.

4.2.2. Property (b). Since $\Psi\left(\frac{1}{h} \mathcal{I}_{\mathscr{C}} \Omega^{\bullet}, 1,1\right)=1$, the assumption $\Psi\left(\mathcal{R}_{\mathscr{X}_{Y}}^{\bullet}, 1,1\right)=1$ for all $Y \in$ $L(\mathscr{X}) \backslash\{V\}$ ensures that $\left.G(Y)\right|_{t=1}=0$ for all $Y \in L(\mathscr{X})$, which gives us property (b).

Remark 4.9. For an hyperplane arrangement $\mathscr{A}$, property (b) is always satisfied as it is proved in [OT92, Proposition 4.132]. The proof of this result relies on the fact that if $\alpha$ is a reduced equation of one of the hyperplanes of $\mathscr{A}$, the complex $\left(\Omega^{\bullet}(\log \mathscr{A}), \frac{\mathrm{d} \alpha}{\alpha} \wedge\right)$ is acyclic. In codimension at least 2 , the modules of multi-logarithmic differential forms are no more stable under the exterior product. In addition, example 5.5 shows that there exist equidimensional reduced subspace arrangements such that this condition is not satisfied. 


\subsubsection{Property (c). Let us generalize [OT92, Proposition 4.134].}

Since $t^{\operatorname{dim}(Y)}$ divides $t^{\ell}$, it is sufficient to prove that $t^{\operatorname{dim}(Y)} \operatorname{divides} \Psi\left(\mathcal{R}_{\mathscr{X}_{Y}}, 1, t\right)$.

We will need the following property, which can be compared with [OT92, Proposition 4.84].

Proposition 4.10. Let $n \in \mathbb{N}, n \neq 0$. Let $\mathscr{Z}$ be an equidimensional subspace arrangement of codimension $k$ in $V_{1}=\mathbb{C}^{n}$ and let $\mathscr{C}_{0}$ be a reduced complete intersection subspace arrangement of codimension $k$ in $\mathbb{C}^{n}$ containing $\mathscr{Z}$. Let $m \in \mathbb{N}, m \geqslant 1$ and $V_{2}=\mathbb{C}^{m}$. Then $\mathscr{C}_{0} \times V_{2}$ is a reduced complete intersection subspace arrangement which contains $\mathscr{Z} \times V_{2}$ and for all $q \in \mathbb{N}$, with $\Omega^{q}\left[V_{2}\right]$ the module of differential forms on $V_{2}$, we have:

$$
\Omega^{q}\left(\log \left(\mathscr{Z} \times V_{2} / \mathscr{C}_{0} \times V_{2}\right)\right)=\sum_{j=0}^{q} \Omega^{j}\left(\log \mathscr{Z} / \mathscr{C}_{0}\right) \otimes \Omega^{q-j}\left[V_{2}\right] .
$$

Proof. We set $S^{1}=\mathbb{C}\left[x_{1}, \ldots, x_{n}\right]$ and $S^{2}=\mathbb{C}\left[y_{1}, \ldots, y_{m}\right]$. We identify any $f \in S^{1}$ with $f \otimes$ $1 \in S^{1} \otimes_{\mathbb{C}} S^{2}$. Let $\left(h_{1}, \ldots, h_{k}\right) \subseteq S^{1}$ be an homogeneous regular sequence defining $\mathscr{C}_{0}$. Then $\left(h_{1} \otimes 1, \ldots, h_{k} \otimes 1\right) \in S^{1} \otimes S^{2}$ is an homogeneous regular sequence which defines the reduced complete intersection $\mathscr{C}^{\prime}=\mathscr{C}_{0} \times V_{2}$.

Let $\omega \in \frac{1}{h} \Omega^{q}\left[V_{1} \times V_{2}\right]$. Then $\omega \in \Omega^{q}\left(\log \left(\mathscr{Z} \times V_{2} / \mathscr{C}^{\prime}\right)\right)$ if and only if for all $f \in \mathcal{I}_{\mathscr{Z}} \otimes S^{2}$, $f \omega \in \frac{1}{h}\left(\mathcal{I}_{\mathscr{C}_{0}} \otimes S^{2}\right) \Omega^{q}\left[V_{1} \times V_{2}\right]$ and $\mathrm{d} f \wedge \omega \in \frac{1}{h}\left(\mathcal{I}_{\mathscr{C}_{0}} \otimes S^{2}\right) \Omega^{q+1}\left[V_{1} \times V_{2}\right]$. Since $\mathcal{I}_{\mathscr{Z}} \otimes S^{2}$ is generated as an ideal in $S^{1} \otimes S^{2}$ by $\mathcal{I}_{\mathscr{Z}} \otimes 1$, it is sufficient to consider only elements in $\mathcal{I}_{\mathscr{Z}} \otimes 1$, which will be denoted by $\mathcal{I}_{\mathscr{Z}}$.

We can write $\omega=\sum_{j=0}^{q} \omega_{j}$ where $\omega_{j} \in \frac{1}{h} \Omega^{j}\left[V_{1}\right] \otimes \Omega^{q-j}\left[V_{2}\right]$. Then $\omega \in \Omega^{q}\left(\log \left(\mathscr{Z} \times V_{2} / \mathscr{C}^{\prime}\right)\right)$ if and only if for all $j \in\{0, \ldots, q\}, \omega_{j} \in \Omega^{q}\left(\log \left(\mathscr{Z} \times V_{2} / \mathscr{C}^{\prime}\right)\right)$.

Let us write $\omega_{j}=\sum_{p=1}^{r} \eta_{p} \wedge \xi_{p}$ where for $1 \leqslant p \leqslant r, \eta_{p} \in \frac{1}{h} \Omega^{j}\left[V_{1}\right]$ and $\xi_{p} \in \Omega^{q-j}\left[V_{2}\right]$ and the $\xi_{p}$ are linearly independant over $\mathbb{C}$. Let us prove as in [OT92, Lemma 4.83] that $\omega_{j} \in \Omega^{q}\left(\log \left(\mathscr{Z} \times V_{2} / \mathscr{C}^{\prime}\right)\right)$ if and only if for all $p, \eta_{p} \in \Omega^{j}\left(\log \mathscr{Z} / \mathscr{C}_{0}\right)$.

Let us assume that for all $p \in\{1, \ldots, q\}, \eta_{p} \in \Omega^{j}\left(\log \mathscr{Z} / \mathscr{C}_{0}\right)$. Then for all $f \in \mathcal{I}_{\mathscr{Z}}, f \eta_{p} \in$ $\frac{1}{h} \mathcal{I}_{\mathscr{C}_{0}} \Omega^{j}\left[V_{1}\right]$ and $\mathrm{d} f \wedge \eta_{p} \in \frac{1}{h} \mathcal{I}_{\mathscr{C}_{0}} \Omega^{j+1}\left[V_{1}\right]$. Therefore, $f\left(\sum \eta_{p} \wedge \xi_{p}\right) \in \frac{1}{h} \mathcal{I}_{\mathscr{C}_{0}} \Omega^{j}\left[V_{1}\right] \otimes \Omega^{q-j}\left[V_{2}\right]$ and $\mathrm{d} f \wedge\left(\sum \eta_{p} \wedge \xi_{p}\right) \in \frac{1}{h} \mathcal{I}_{\mathscr{C}_{0}} \Omega^{j+1}\left[V_{1}\right] \otimes \Omega^{q-j}\left[V_{2}\right]$ and $\omega_{j} \in \Omega^{q}\left(\log \left(\mathscr{Z} \times V_{2} / \mathscr{C}^{\prime}\right)\right)$. Thus $\omega \in \Omega^{q}(\log (\mathscr{Z} \times$ $\left.\left.V_{2} / \mathscr{C}^{\prime}\right)\right)$.

Let us assume now that $\omega \in \Omega^{q}\left(\log \left(\mathscr{Z} \times V_{2} / \mathscr{C}^{\prime}\right)\right)$, or equivalently that for all $j \in\{0, \ldots, q\}$, $\omega_{j} \in \Omega^{q}\left(\log \left(\mathscr{Z} \times V_{2} / \mathscr{C}^{\prime}\right)\right)$. Let $f \in \mathcal{I}_{\mathscr{Z}}$. We have

$$
\begin{gathered}
f\left(\sum \eta_{p} \wedge \xi_{p}\right)=\sum\left(f \eta_{p}\right) \wedge \xi_{p} \in \frac{1}{h} \mathcal{I}_{\mathscr{C}_{0}} \Omega^{j}\left[V_{1}\right] \otimes \Omega^{q-j}\left[V_{2}\right] \\
\mathrm{d} f \wedge\left(\sum \eta_{p} \wedge \xi_{p}\right)=\sum_{p}\left(\mathrm{~d} f \wedge \eta_{p}\right) \wedge \xi_{p} \in \frac{1}{h} \mathcal{I}_{\mathscr{C}_{0}} \Omega^{j+1}\left[V_{1}\right] \otimes \Omega^{q-j}\left[V_{2}\right] .
\end{gathered}
$$

Therefore, since the $\xi_{p}$ are linearly independant, we have for all $p, f \eta_{p} \in \frac{1}{h} \mathcal{I}_{\mathscr{C}_{0}} \Omega^{j}\left[V_{1}\right]$ and $\mathrm{d} f \wedge \eta_{p} \in$ $\frac{1}{h} \mathcal{I}_{\mathscr{C}_{0}} \Omega^{j+1}\left[V_{1}\right]$ so that $\eta_{p} \in \Omega^{j}\left(\log \mathscr{Z} / \mathscr{C}_{0}\right)$. Hence the result.

Let $Y \in L(\mathscr{X}), Y \neq \mathbb{C}^{\ell}$. Let $m$ be the dimension of $Y$. Then there exists $u \in\{1, \ldots, s\}$ and $1 \leqslant i_{1}<\ldots<i_{u} \leqslant s$ such that $\mathscr{X}_{Y}=\mathscr{X}_{i_{1}} \cup \cdots \cup \mathscr{X}_{i_{u}}$. Since all the irreducible components of $\mathscr{X}_{Y}$ contains $Y$, there exists an equidimensional subspace arrangement $\mathscr{Z}=\mathscr{Z}_{1} \cup \cdots \cup \mathscr{Z}_{u}$ of codimension $k$ in $\mathbb{C}^{\ell-m}$ such that $\mathscr{X}_{Y}=\mathscr{Z} \times Y=\left(\mathscr{Z}_{1} \times Y\right) \cup \cdots \cup\left(\mathscr{Z}_{u} \times Y\right)$. Let $\mathscr{C}_{0}$ be a reduced complete intersection subspace arrangement of codimension $k$ in $\mathbb{C}^{\ell-m}$ containing $\mathscr{Z}$.

Let us consider $\Psi\left(\Omega^{\bullet}\left(\log \left(\mathscr{Z} \times Y / \mathscr{C}_{0} \times Y\right)\right), x, t\right)$. One can notice that this function may be different from $\Psi\left(\Omega^{\bullet}(\log (\mathscr{Z} \times Y / \mathscr{C})), x, t\right)$ since $\mathscr{C}$ and $\mathscr{C}^{\prime}:=\mathscr{C}_{0} \times Y$ may be different. However, since the modules of multi-residues are intrinsic, the $\Psi$-function relative to $\mathcal{R}_{\mathscr{Z} \times Y}^{\bullet}$ does not depend on the choice of the complete intersection. 
Let $q \in \mathbb{N}$. Since $\Omega^{q}\left(\log \mathscr{X}_{Y} / \mathscr{C}^{\prime}\right)=\sum_{j=0}^{q} \Omega^{j}\left(\log \mathscr{Z} / \mathscr{C}_{0}\right) \otimes \Omega^{q-j}[Y]$, we have:

$$
\operatorname{Poin}\left(\Omega^{q}\left(\log \mathscr{X}_{Y} / \mathscr{C}^{\prime}\right), x\right)=\sum_{j=0}^{q} \operatorname{Poin}\left(\Omega^{j}\left(\log \mathscr{Z} / \mathscr{C}_{0}\right), x\right) \operatorname{Poin}\left(\Omega^{q-j}[Y], x\right)
$$

Therefore, we have:

$$
\begin{aligned}
\Psi\left(\Omega^{\bullet}\left(\log \mathscr{X}_{Y} / \mathscr{C}^{\prime}\right), x, t\right) & =\sum_{q=0}^{\ell} \sum_{j=0}^{q} \operatorname{Poin}\left(\Omega^{j}\left(\log \mathscr{Z} / \mathscr{C}_{0}\right), x\right)(t(1-x)-1)^{j} \operatorname{Poin}\left(\Omega^{q-j}[Y], x\right)(t(1-x)-1)^{q-j} \\
& =\left(\sum_{p=0}^{\ell-m} \operatorname{Poin}\left(\Omega^{p}\left(\log \mathscr{Z} / \mathscr{C}_{0}\right), x\right)(t(1-x)-1)^{p}\right) \cdot\left(\sum_{q=0}^{m} \operatorname{Poin}\left(\Omega^{q}[Y], x\right)(t(1-x)-1)^{q}\right) \\
& =\Psi\left(\Omega^{\bullet}\left(\log \mathscr{Z} / \mathscr{C}_{0}\right), x, t\right) \cdot \Psi\left(\Omega^{\bullet}[Y], x, t\right)
\end{aligned}
$$

By [OT92, Proposition 4.131], we have $\Psi\left(\Omega^{\bullet}[Y], x, t\right)=t^{m}$ since for all $q \in \mathbb{N}$, the module of logarithmic forms ${ }^{3}$ of the empty arrangement in $Y$ is $\Omega^{q}[Y]$. Therefore, $t^{m} \operatorname{divides} \Psi\left(\Omega^{\bullet}\left(\log \mathscr{X}_{Y} / \mathscr{C}^{\prime}\right), x, t\right)$. We deduce from propositions 3.11 and 3.10 that $t^{m}$ also divides $\Psi\left(\mathcal{R}_{\mathscr{X}_{Y}}^{\bullet}, x, t\right)$, so that $t^{m}$ divides $\widetilde{\Psi}\left(\mathscr{X}_{Y}, x, t\right)$. Therefore, $t^{m}$ divides $G(Y)$ which gives us property (c).

4.2.4. Property (d). Our purpose is to prove that for all $Y \in L(\mathscr{X})$,

$$
\operatorname{deg}_{t}\left(\sum_{Z \in L_{Y}} \mu(Z, Y) G(Z)\right) \leqslant \operatorname{dim} Y
$$

where $L_{Y}=\{Z \in L(\mathscr{X}) ; Z \leqslant Y\}$.

Since for all $Z \in L(\mathscr{X}), G(Z)=\Psi\left(\frac{1}{h} \mathcal{I}_{\mathscr{C}} \Omega^{\bullet}, 1, t\right)-\Psi\left(\mathcal{R}_{\mathscr{X}_{Z}}^{\bullet}, 1, t\right)$ where $\Psi\left(\frac{1}{h} \mathcal{I}_{\mathscr{C}} \Omega^{\bullet}, 1, t\right)$ does not depend on $Z$, and since by [OT92, Lemma 2.38], $\sum_{Z \in L_{Y}} \mu(Z, Y)=0$, we have

$$
\sum_{Z \in L_{Y}} \mu(Z, Y) G(Z)=\sum_{Z \in L_{Y}} \mu(Z, Y) \Psi\left(\mathcal{R}_{\mathscr{X}_{Z}}^{\bullet}, 1, t\right)=\sum_{Z \in L_{Y}} \mu(Z, Y) \Psi\left(\Omega^{\bullet} \log \left(\mathscr{X}_{Z} / \mathscr{C}\right), 1, t\right) .
$$

Therefore, it is equivalent to prove that property (d) is satisfied for the modules of multilogarithmic forms.

Notation 4.11. Let $Y \in L(\mathscr{X})$ and $\mathfrak{p} \in \operatorname{Spec}(S)$. We recall that $\mathscr{X}_{Y}$ denotes the subarrangement composed of the components of $\mathscr{X}$ which contain $Y$. We set

$$
Y(\mathfrak{p})=\bigcap_{\substack{\mathscr{X}_{i} \in \mathscr{X}_{Y} \\ \mathcal{I}_{\mathscr{X}_{i}} \subseteq \mathfrak{p}}} \mathscr{X}_{i}
$$

We then have $Y \subseteq Y(\mathfrak{p})$, so that $\mathscr{X}_{Y(\mathfrak{p})} \subseteq \mathscr{X}_{Y}$.

Definition 4.12 ([OT92, Definition 4.121]). A covariant functor $F: L(\mathscr{X}) \rightarrow(S$-mod $)$ is called local if for all $\mathfrak{p} \in \operatorname{Spec}(S)$ and for all $Y \in L(\mathscr{X})$, the localization at $\mathfrak{p}$ of the map $\nu_{Y(\mathfrak{p}), Y}: F(Y(\mathfrak{p})) \rightarrow$ $F(Y)$ is an isomorphism.

The following proposition is given for hyperplane arrangements, but it can be proved for subspace arrangements with exactly the same proof.

Proposition 4.13 ([OT92, Theorem 4.128]). Let $Y \in L(\mathscr{X})$. If $F: L(\mathscr{X}) \rightarrow(S$-mod $)$ is a local covariant functor, then

$$
\sum_{Z \in L_{Y}} \mu(Z, Y) \operatorname{Poin}(F(Z), x)
$$

has a pole at $x=1$ of order at most $\operatorname{dim} Y$.

Let us prove the following proposition:

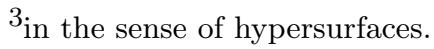


Proposition 4.14. Let $q \in \mathbb{N}$. The functor $F_{q}: L(\mathscr{X}) \rightarrow(S$-mod $)$ defined for $Y \in L(\mathscr{X})$ by $F_{q}(Y)=\Omega^{q}\left(\log \mathscr{X}_{Y} / \mathscr{C}\right)$ is a local covariant functor.

Proof. Let us prove first that $F_{q}$ is a covariant functor. Let $Y_{1} \leqslant Y_{2}$. Then $\mathscr{X}_{Y_{1}} \subseteq \mathscr{X}_{Y_{2}}$, so that $\mathcal{I}_{\mathscr{X}_{Y_{2}}} \subseteq \mathcal{I}_{\mathscr{X}_{Y_{1}}}$. From the definition of the modules of multi-logarithmic forms, it is easy to see that $\Omega^{q}\left(\log \mathscr{X}_{Y_{1}} / \mathscr{C}\right) \subseteq \Omega^{q}\left(\log \mathscr{X}_{Y_{2}} / \mathscr{C}\right)$, which shows that the functor $F_{q}$ is covariant.

Let us prove that the functor $F_{q}$ is local. Let $\mathfrak{p} \in \operatorname{Spec}(S)$.

Let $Y \in L(\mathscr{X})$. Since $Y(\mathfrak{p}) \leqslant Y$, we have $\Omega^{q}\left(\log \mathscr{X}_{Y(\mathfrak{p})} / \mathscr{C}\right) \subseteq \Omega^{q}\left(\log \mathscr{X}_{Y} / \mathscr{C}\right)$. Since localization is an exact functor, we have:

$$
\left(\Omega^{q}\left(\log \mathscr{X}_{Y(\mathfrak{p})} / \mathscr{C}\right)\right)_{\mathfrak{p}} \subseteq\left(\Omega^{q}\left(\log \mathscr{X}_{Y} / \mathscr{C}\right)\right)_{\mathfrak{p}}
$$

Let us prove that we have an equality. If $\mathscr{X}_{Y(\mathfrak{p})}=\mathscr{X}_{Y}$, the equality is clear. Let us assume that $\mathscr{X}_{Y(\mathfrak{p})} \neq \mathscr{X}_{Y}$. Let us denote by $\mathscr{X}^{\prime}$ the union of the components of $\mathscr{X}_{Y}$ which are not in $\mathscr{X}_{Y(\mathfrak{p})}$. In particular, $\mathcal{I}_{\mathscr{X}^{\prime}} \nsubseteq \mathfrak{p}$. Let $Q \in \mathcal{I}_{\mathscr{X}^{\prime}}$ be such that $Q \notin \mathfrak{p}$. Then for all component $\mathscr{X}_{i}$ of $\mathscr{X}_{Y(\mathfrak{p})}$, $Q \notin \mathcal{I}_{\mathscr{X}_{i}}$.

Let $\omega \in \Omega^{q}\left(\log \mathscr{X}_{Y} / \mathscr{C}\right)$. Let us prove that $Q \omega \in \Omega^{q}\left(\log \mathscr{X}_{Y(\mathfrak{p})} / \mathscr{C}\right)$, which will give us the equality in (9).

Let $c_{\mathscr{X}_{Y} / \mathscr{C}}$ be the fundamental form of $\mathscr{X}_{Y}$ (see definition 2.8). Let us denote by $\mathscr{Z}$ the union of the components of $\mathscr{C}$ which are not in $\mathscr{X}_{Y}$. By theorem 2.10, there exists $g \in S$ inducing a non zero divisor in $\mathcal{O}_{\mathscr{C}}, \xi \in \Omega^{q-k}$ and $\eta \in \frac{1}{h} \mathcal{I}_{\mathscr{C}} \Omega^{q}$ such that:

$$
g \omega=\frac{c_{\mathscr{X}_{Y} / \mathscr{C}}}{h} \wedge \xi+\eta .
$$

In particular, from the definition of $c_{\mathscr{X}_{Y} / \mathscr{C}}$ and since $g$ induces a non zero divisor in $\mathcal{O}_{\mathscr{C}}$, one can see that $\omega \in \frac{1}{h} \mathcal{I}_{\mathscr{Z}} \Omega^{q}$. Let $\mathscr{Z}^{\prime}=\mathscr{Z} \cup \mathscr{X}^{\prime}$. In particular, $\mathscr{C}=\mathscr{Z}^{\prime} \cup \mathscr{X}_{Y(\mathfrak{p})}$. We have $Q \omega \in \frac{1}{h} \mathcal{I}_{\mathscr{Z}} \Omega^{q}$ so that $\mathcal{I}_{\mathscr{X}_{Y(\mathfrak{p})}} Q \omega \subseteq \frac{1}{h} \mathcal{I}_{\mathscr{C}} \Omega^{q}$. Let $f \in \mathcal{I}_{\mathscr{X}_{Y(\mathfrak{p})}}$. Let us prove that $\mathrm{d} f \wedge Q \omega \in \frac{1}{h} \mathcal{I}_{\mathscr{C}} \Omega^{q+1}$. Since $f Q \in \mathcal{I}_{\mathscr{X}_{Y}}$ and $\omega \in \Omega^{q}\left(\log \mathscr{X}_{Y} / \mathscr{C}\right)$, we have $\mathrm{d}(f Q) \wedge \omega \in \frac{1}{h} \mathcal{I}_{\mathscr{C}} \Omega^{q}$. We thus have, since $\mathcal{I}_{\mathscr{C}} \subseteq \mathcal{I}_{\mathscr{X}_{Y(\mathfrak{p})}}$ :

$$
\mathrm{d} f \wedge Q \omega=\mathrm{d}(f Q) \wedge \omega-f \mathrm{~d} Q \wedge \omega \in \frac{1}{h} \mathcal{I}_{\mathscr{X}_{Y(\mathfrak{p})}} \Omega^{q+1} .
$$

Since in addition $Q \omega \in \frac{1}{h} \mathcal{I}_{\mathscr{Z}} \Omega^{q}$, and $\mathcal{I}_{\mathscr{C}}=\mathcal{I}_{\mathscr{Z}} \cap \mathcal{I}_{\mathscr{X}_{Y(\mathfrak{p})}}$, we have $\mathrm{d} f \wedge Q \omega \in \frac{1}{h} \mathcal{I}_{\mathscr{C}} \Omega^{q+1}$. Therefore, $Q \omega \in \Omega^{q}\left(\log \left(\mathscr{X}_{Y(\mathfrak{p})} / \mathscr{C}\right)\right)$. Since $Q \notin \mathfrak{p}$, it shows that we have

$$
\left(\Omega^{q}\left(\log \left(\mathscr{X}_{Y(\mathfrak{p})} / \mathscr{C}\right)\right)_{\mathfrak{p}}=\left(\Omega^{q}\left(\log \left(\mathscr{X}_{Y} / \mathscr{C}\right)\right)\right)_{\mathfrak{p}} .\right.
$$

We can now prove property (d).

We have:

$$
\begin{aligned}
\sum_{Z \in L_{Y}} \mu(Z, Y) G(Z) & =\sum_{Z \in L_{Y}} \mu(Z, Y) \Psi\left(\Omega^{\bullet} \log \left(\mathscr{X}_{Z} / \mathscr{C}\right), 1, t\right) \\
& =\sum_{Z \in L_{Y}} \sum_{q=0}^{\ell} \mu(Z, Y) \operatorname{Poin}\left(\left.\Omega^{q}\left(\log \left(\mathscr{X}_{Z} / \mathscr{C}\right), x\right)(t(1-x)-1)^{q}\right|_{x=1}\right. \\
& =\sum_{q=0}^{\ell}\left(\left.\sum_{Z \in L_{Y}} \mu(Z, Y) \operatorname{Poin}\left(\Omega^{q}\left(\log \left(\mathscr{X}_{Z} / \mathscr{C}\right), x\right)\right)(t(1-x)-1)^{q}\right|_{x=1}\right. \\
& =\sum_{q=0}^{\ell} M_{q}(x)(t(1-x)-1)^{q}
\end{aligned}
$$

where $M_{q}(x)=\sum_{Z \in L_{Y}} \mu(Z, Y) \operatorname{Poin}\left(\Omega^{q}\left(\log \left(\mathscr{X}_{Z} / \mathscr{C}\right), x\right)=\sum_{Z \in L_{Y}} \mu(Z, Y) \operatorname{Poin}\left(F_{q}(Z), x\right)\right.$. 
By propositions 4.13 and 4.14, $(1-x)^{\operatorname{dim}(Y)} M_{q}(x)$ has no pole at $x=1$. As in the proof of [OT92, Theorem 4.136 (4)], we deduce that for all $n>\operatorname{dim}(Y)$, the coefficient of $t^{n}$ in $M_{q}(x)(t(1-x)-1)^{q}$ lies in $(1-x) \mathbb{Z}\left[x, x^{-1}\right]$. Hence the result.

\section{EXAMPLES}

We give in this section several examples. We first show that any line arrangement satisfies the generalized Solomon-Terao formula. We then give an example of surface in $\mathbb{C}^{4}$ which does not satisfy the formula.

5.1. Case of line arrangements. We already considered the case of complete intersection line arrangements in proposition 3.13. Let us prove that any line arrangement in $\mathbb{C}^{\ell}$ satisfies the condition of theorem 4.2, so that the generalization of Solomon Terao formula holds for any line arrangement.

Corollary 5.1. For any line arrangement $\mathscr{X}$ in $\mathbb{C}^{\ell}$,

$$
\chi(\mathscr{X}, t)=t^{\ell}-\Psi\left(\mathcal{R}_{\mathscr{X}}^{\bullet}, 1, t\right) .
$$

Proof. If $\mathscr{X}$ is composed of only one line, then $\mathscr{X}$ is smooth and we have $\mathcal{R}_{\mathscr{X}}=\mathcal{O}_{\mathscr{X}}$ and $\mathcal{R}_{\mathscr{X}}^{1}=\mathcal{O}_{\mathscr{X}}$. Therefore,

$$
\Psi\left(\mathcal{R}_{\mathscr{X}}^{\bullet}, x, t\right)=\operatorname{Poin}\left(\mathcal{O}_{\mathscr{X}}, x, t\right)+(t(1-x)-1) \operatorname{Poin}\left(\mathcal{O}_{\mathscr{X}}, x, t\right)=t(1-x) \operatorname{Poin}\left(\mathcal{O}_{\mathscr{X}}, x, t\right) .
$$

In addition, $\operatorname{Poin}\left(\mathcal{O}_{\mathscr{X}}, x\right)=\frac{1}{1-x}$. Therefore, $t^{\ell}-\Psi\left(\mathcal{R}_{\mathscr{X}}^{\bullet}, 1, t\right)=t^{\ell}-t=\chi(\mathscr{X}, t)$.

From now on, we will assume that $\mathscr{X}$ has at least two components. In particular, it means that $\mathscr{X}$ is singular.

Thanks to theorem 4.2 , it is sufficient to prove that for any line arrangement $\mathscr{X}$ in $\mathbb{C}^{\ell}$ we have $\Psi\left(\mathcal{R}_{\mathscr{X}}^{\bullet}, 1,1\right)=1$. We will in fact prove that $\Psi\left(\mathcal{R}_{\mathscr{X}}^{\bullet}, x, 1\right)=1$.

Let us denote $\mathscr{C}=\mathscr{X} \cup \mathscr{Y}$ where $\mathscr{Y}$ is the union of the irreducible components of $\mathscr{C}$ which are not in $\mathscr{X}$.

By remark 2.17, we have an inclusion $\mathcal{R}_{\mathscr{X}} \hookrightarrow \mathcal{R}_{\mathscr{C}}$. Thanks to this inclusion, we will consider $\mathcal{R}_{\mathscr{X}}$ as a submodule of $\mathcal{R}_{\mathscr{C}}$. More precisely, $\mathcal{R}_{\mathscr{X}}$ can be identified with $\left\{\rho \in \mathcal{R}_{\mathscr{C}} ;\left.\rho\right|_{\mathscr{Y}}=0\right\}$ (see [Pol16b, Proposition 4.2.7]).

Let $\operatorname{Jac}\left(h_{1}, \ldots, h_{\ell-1}\right)$ be the Jacobian matrix associated with $\left(h_{1}, \ldots, h_{\ell-1}\right)$. For $i \in\{1, \ldots, \ell\}$ we denote by $J_{i}$ the minor $(\ell-1) \times(\ell-1)$ of $\operatorname{Jac}\left(h_{1}, \ldots, h_{\ell-1}\right)$ obtained by removing the $i$ th column. Let $c_{1}, \ldots, c_{\ell} \in \mathbb{C}$ be such that $g=c_{1} J_{1}+\ldots+c_{\ell} J_{\ell} \in S$ and $y=\sum_{i=1}^{\ell}(-1)^{i-1} c_{i} x_{i} \in S$ induce non zero divisors of $\mathcal{O}_{\mathscr{C}}$.

By [Pol16b, Proposition 6.1.24], the module $\mathcal{R}_{\mathscr{C}}$ is generated by $1=\frac{g}{g}$ and $\frac{y}{g}$.

In addition, for all $i, j \in\{1, \ldots, \ell\}$ we have (see [Pol16b, (6.13)]):

$$
(-1)^{j-1} x_{j} J_{i}=(-1)^{i-1} x_{i} J_{j} \bmod \mathcal{I}_{\mathscr{C}} .
$$

We then have for all $j \in\{1, \ldots, \ell\}$ :

$$
x_{j} g=(-1)^{j-1} J_{j} y \bmod \mathcal{I}_{\mathscr{C}} .
$$

Therefore, for all $\rho \in \mathcal{R}_{\mathscr{C}}$, there exists $a \in \mathcal{O}_{\mathscr{C}}$ and $c \in \mathbb{C}$ such that $\rho=a \frac{y}{g}+c$.

We first prove the following lemmas.

Lemma 5.2. There exists $a_{1} \in \mathcal{O}_{\mathscr{C}}$ and $c_{1} \in \mathbb{C}, c_{1} \neq 0$ such that $\rho_{1}=a_{1} \frac{y}{g}+c_{1} \in \mathcal{R}_{\mathscr{X}} \subseteq \mathcal{R}_{\mathscr{C}}$.

Proof. Let us assume that for all $\rho=a \frac{y}{g}+c \in \mathcal{R}_{\mathscr{X}}$, we have $c=0$. Then it means that $\mathcal{R}_{\mathscr{X}}$ is contained in the $\mathcal{O}_{\mathscr{C}}$-module generated by $\frac{y}{g}$.

Let us consider the value map on $\mathscr{C}$ as in [Pol17, Definition 2.1]. Let us denote by $p$ the number of irreducible components of $\mathscr{C}$. We still assume that $\mathscr{X}$ has at least two components, so that $p \geqslant 2$.

The value map val associates with any element $f \in \operatorname{Frac}\left(\mathcal{O}_{\mathscr{C}}\right)$ the $p$-uple of its valuation along each irreducible component of $\mathscr{C}$. We denote $\mathscr{C}=\mathscr{X}_{1} \cup \cdots \cup \mathscr{X}_{s} \cup \mathscr{Y}_{s+1} \cup \cdots \cup \mathscr{Y}_{p}$ where the $\mathscr{X}_{i}$ are the irreducible components of $\mathscr{X}$. For all $a, b \in \operatorname{Frac}\left(\mathcal{O}_{\mathscr{C}}\right)$, we have $\operatorname{val}(a b)=\operatorname{val}(a)+\operatorname{val}(b)$.

\footnotetext{
${ }^{4}$ Since $\mathcal{R}_{\mathscr{X}}$ is graded, we may assume that $\rho_{1}$ is an homogeneous element.
} 
If $I \subseteq \operatorname{Frac}\left(\mathcal{O}_{\mathscr{C}}\right)$ is an ideal, we set $\operatorname{val}(I)=\{\operatorname{val}(g) ; g \in I\} \cap \mathbb{Z}^{p}$.

We denote by $\mathcal{O}_{\widetilde{\mathscr{C}}} \subseteq \operatorname{Frac}\left(\mathcal{O}_{\mathscr{C}}\right)$ the normalization of $\mathcal{O}_{\mathscr{C}}$. In particular, since $\mathscr{C}$ is the union of $p$ lines, $\mathcal{O}_{\tilde{\mathscr{C}}} \simeq \bigoplus_{i=1}^{p} \mathbb{C}\left[t_{i}\right]$. In addition, by [Pol16b, Proposition 3.1.28], $\mathcal{O}_{\tilde{\mathscr{C}}} \subseteq \mathcal{R}_{\mathscr{C}}$. We denote by $\gamma=\left(\gamma_{1}, \ldots, \gamma_{p}\right) \in \mathbb{N}^{p}$ the conductor of $\mathscr{C}$, which is the lowest element for the product order in $\mathbb{Z}^{p}$ which satisfies $\gamma+\mathbb{N}^{p} \subseteq \operatorname{val}\left(\mathcal{O}_{\mathscr{C}}\right)$ (see [Pol17, Lemma 2.8]).

Let $\theta \in \mathcal{O}_{\widetilde{\mathscr{C}}} \subseteq \mathcal{R}_{\mathscr{C}}$ be such that $\left.\theta\right|_{\mathscr{X}_{1}}=1$ and for all $i \in\{2, \ldots, s\}$, for all $j \in\{s+1, \ldots, p\}$, $\left.\theta\right|_{\mathscr{X}_{i}}=0$ and $\left.\theta\right|_{\mathscr{Y}_{j}}=0$. Then $\theta \in \mathcal{R}_{\mathscr{X}}$ since $\left.\theta\right|_{\mathscr{Y}}=0$. Thus, by assumption, there exists $a \in \mathcal{O}_{\mathscr{C}}$ such that $\theta=a \frac{y}{g}$. In particular, val $\left(a \frac{y}{g}\right)=(0, \infty, \ldots, \infty)$.

By [Pol16b, Lemma 6.1.22], we have val $\left(\frac{y}{g}\right)=-\gamma+\underline{1}$. Let $b \in \mathcal{O}_{\mathscr{C}}$ be such that $\operatorname{val}(b)=\gamma$. Then val $\left(b \frac{y}{g}\right)=(1, \ldots, 1)$. Therefore, using [Pol17, Proposition 2.10], we have val $\left((a+b) \frac{y}{g}\right)=$ $(0,1, \ldots, 1)$. Since val $\left((a+b) \frac{y}{g}\right)=\operatorname{val}(a+b)+\operatorname{val}\left(\frac{y}{g}\right)$, we have $\operatorname{val}(a+b)=\left(\gamma_{1}-1, \gamma_{2}, \ldots, \gamma_{p}\right) \in$ $\operatorname{val}\left(\mathcal{O}_{\mathscr{C}}\right)$

However, the set $\left\{v \in \operatorname{val}\left(\mathcal{O}_{\mathscr{C}}\right) ; v_{1}=\gamma_{1}-1\right.$ and $\left.\forall j \neq 1, v_{j} \geqslant \gamma_{j}\right\}$ is empty (see [Pol17, Proposition 2.17]).

Hence the result.

Lemma 5.3. The module $\mathcal{R}_{\mathscr{X}}$ is generated as an $\mathcal{O}_{\mathscr{C}}$-module and as an $\mathcal{O}_{\mathscr{X}}$-module by the elements in $\mathcal{I}_{\mathscr{Y}} \frac{y}{g}$ and the residue $\rho_{1}$ introduced in lemma 5.2.

Proof. By [Pol16b, Proposition 4.2.7], for all $\rho \in \mathcal{R}_{\mathscr{C}}$ we have $\rho \in \mathcal{R}_{\mathscr{X}}$ if and only if the restriction of $\rho$ to $\mathscr{Y}$ is zero. Therefore, we have $\mathcal{I}_{\mathscr{Y}} \frac{y}{g} \subseteq \mathcal{R}_{\mathscr{X}}$.

Let $\rho \in \mathcal{R}_{\mathscr{X}}$. We denote by $\overline{\mathcal{I}_{\mathscr{Y}}} \subseteq \mathcal{O}_{\mathscr{C}}$ the image of $\mathcal{I}_{\mathscr{Y}}$ in $\mathcal{O}_{\mathscr{C}}$. There exists $a \in \mathcal{O}_{\mathscr{C}}$ and $c \in \mathbb{C}$ such that $\rho=a \frac{y}{g}+c$. We thus have:

$$
a y+c g \in \overline{\mathcal{I}} \mathscr{Y} .
$$

We have:

$$
\begin{aligned}
a y+c g & =a y+\frac{c}{c_{1}}\left(a_{1} y+c_{1} g\right)-\frac{c}{c_{1}} a_{1} y \\
& =\left(a-\frac{c}{c_{1}} a_{1}\right) y+\frac{c}{c_{1}}\left(a_{1} y+c_{1} g\right) \in \overline{\mathcal{I}_{\mathscr{Y}}}
\end{aligned}
$$

Since $\frac{a_{1} y+c_{1} g}{g} \in \mathcal{R}_{\mathscr{X}}$, we have $a_{1} y+c_{1} g \in \overline{\mathcal{I}_{\mathscr{Y}}}$ and therefore $\left(a-\frac{c}{c_{1}} a_{1}\right) y \in \overline{\mathcal{I}_{\mathscr{Y}}}$. Since $y$ induces a non zero divisor in $\mathcal{O}_{\mathscr{C}}$, we have $a-\frac{c}{c_{1}} a_{1} \in \overline{\mathcal{I}_{\mathscr{Y}}}$. Thus, since $\mathcal{I}_{\mathscr{C}} \subseteq \mathcal{I}_{\mathscr{Y}}$, we have:

$$
\rho=\mu \frac{y}{g}+\frac{c}{c_{1}} \rho_{1}
$$

with $\mu \in \mathcal{I}_{\mathscr{Y}}$.

Let us prove corollary 5.1. We have $\mathcal{R}_{\mathscr{X}}=\frac{1}{g}\left(\mathcal{I}_{\mathscr{Y}} \frac{y}{g}+\mathcal{O}_{\mathscr{C}} \frac{a_{1} y+c_{1} g}{g}\right)$. We have the following exact sequence:

$$
0 \rightarrow \overline{\mathcal{I}_{\mathscr{Y}}} \stackrel{y}{\rightarrow} y \overline{\mathcal{I}_{\mathscr{Y}}}+\left(a_{1} y+c_{1} g\right) \mathcal{O}_{\mathscr{C}} \rightarrow \frac{\left(a_{1} y+c_{1} g\right) S+y \mathcal{I}_{\mathscr{Y}}+\mathcal{I}_{\mathscr{C}}}{y \mathcal{I}_{\mathscr{Y}}+\mathcal{I}_{\mathscr{C}}} \rightarrow 0 .
$$

Let us compute a free resolution of the module $M=\frac{\left(a_{1} y+c_{1} g\right) S+y \mathcal{I}_{\mathscr{Y}}+\mathcal{I}_{\mathscr{C}}}{y \mathcal{I}_{\mathscr{Y}}+\mathcal{I}_{\mathscr{C}}}$. We have the following exact sequence:

$$
0 \rightarrow\left(\left(y \mathcal{I}_{\mathscr{Y}}+\mathcal{I}_{\mathscr{C}}\right):\left(a_{1} y+c_{1} g\right)\right)_{S} \rightarrow S \stackrel{\left(a_{1} y+c_{1} g\right)}{\longrightarrow} M \rightarrow 0
$$

Let us compute $\mathcal{T}:=\left(\left(y \mathcal{I}_{\mathscr{Y}}+\mathcal{I}_{\mathscr{C}}\right):\left(a_{1} y+c_{1} g\right)\right)_{S}$. Let $i \in\{1, \ldots, \ell\}$. Let us prove that $x_{i} \in \mathcal{T}$. We have by (10):

$$
\begin{aligned}
x_{i}\left(a_{1} y+c_{1} g\right) & =x_{i} a_{1} y+c_{1} x_{i} g \\
& =\left(x_{i} a_{1}+(-1)^{i-1} J_{i}\right) y+\lambda \in \mathcal{I}_{\mathscr{Y}}
\end{aligned}
$$


with $\lambda \in \mathcal{I}_{\mathscr{C}}$. Since $\mathcal{I}_{\mathscr{C}} \subseteq \mathcal{I}_{\mathscr{Y}}$ and $y$ is a non zero divisor in $\mathcal{O}_{\mathscr{C}}$, we have $\left(x_{i} a_{1}+(-1)^{i-1} J_{i}\right) \in \mathcal{I}_{\mathscr{Y}}$, so that $x_{i}\left(a_{1} y+c_{1} g\right) \in y \mathcal{I}_{\mathscr{Y}}+\mathcal{I}_{\mathscr{C}}$ and $x_{i} \in \mathcal{T}$. Therefore, $\left(x_{1}, \ldots, x_{\ell}\right) \subseteq \mathcal{T}$.

In addition, from lemma 5.2 , we have $\left(a_{1} y+c_{1} g\right) \notin y \overline{\mathcal{I}_{\mathscr{Y}}}$, so that we have $\left\langle x_{1}, \ldots, x_{\ell}\right\rangle=\mathcal{T}$. Then a minimal free resolution of $\left(\left(y \mathcal{I}_{\mathscr{Y}}+\mathcal{I}_{\mathscr{C}}\right):\left(a_{1} y+c_{1} g\right)\right)_{S}$ is deduced from the Koszul complex associated with the regular sequence $\left(x_{1}, \ldots, x_{\ell}\right)$ :

$$
0 \rightarrow S(-(d-\ell+1)-\ell)^{\left(\begin{array}{l}
\ell \\
\ell
\end{array}\right)} \rightarrow \cdots \rightarrow S(-(d-\ell+1)-1)^{\left(\begin{array}{l}
\ell \\
1
\end{array}\right)} \rightarrow S(-(d-\ell+1))^{\left(\begin{array}{l}
\ell \\
0
\end{array}\right)} \rightarrow M \rightarrow 0 .
$$

From the exact sequence (12) and the additivity of Poincare series, we have:

$$
\operatorname{Poin}\left(y \overline{\mathcal{I}_{\mathscr{C}}}+\left(a_{1} y+c_{1} g\right) \mathcal{O}_{\mathscr{C}}, x\right)=x \operatorname{Poin}\left(\overline{\mathcal{I}_{\mathscr{Y}}}, x\right)+\operatorname{Poin}(M, x) .
$$

Since $\mathcal{R}_{\mathscr{X}}=\frac{1}{g}\left(y \overline{\mathcal{I} \mathscr{Y}}+\left(a_{1} y+c_{1} g\right) \mathcal{O}_{\mathscr{C}}\right)$, we have:

$$
\operatorname{Poin}\left(\mathcal{R}_{\mathscr{X}}, x\right)=x^{\ell-d} \operatorname{Poin}\left(\overline{\mathcal{I}_{\mathscr{Y}}}, x\right)+x^{\ell-d-1} \operatorname{Poin}(M, x) .
$$

From the free resolution (13), we have $\operatorname{Poin}(M, x)=x^{d-\ell+1} \sum_{i=0}^{\ell}\left(\begin{array}{l}\ell \\ i\end{array}\right) \frac{x^{i}}{(1-x)^{\ell}}=x^{d-\ell+1}$. Therefore,

$$
\operatorname{Poin}\left(\mathcal{R}_{\mathscr{X}}, x\right)=x^{\ell-d} \operatorname{Poin}\left(\overline{\mathcal{I}_{\mathscr{Y}}}, x\right)+1 .
$$

Let us consider $\mathcal{R}_{\mathscr{X}}^{1}$. We have $\Omega^{\ell}(\log \mathscr{C})=\frac{1}{h} \Omega^{\ell}$. From definition 2.5, we deduce that

$$
\Omega^{\ell}(\log \mathscr{X} / \mathscr{C})=\frac{1}{h} \mathcal{I}_{\mathscr{Y}} \Omega^{\ell} .
$$

Therefore, $\mathcal{R}_{\mathscr{X}}^{1}=\mathcal{I}_{\mathscr{Y}} \operatorname{res}_{\mathscr{C}}\left(\frac{\mathrm{d} x_{1} \wedge \cdots \wedge \mathrm{d} x_{\ell}}{h}\right) \simeq \overline{\mathcal{I}_{\mathscr{Y}}}$. In addition, res $\mathscr{C}\left(\frac{\mathrm{d} x_{1} \wedge \cdots \wedge \mathrm{d} x_{\ell}}{h}\right)$ is homogeneous of degree $-(d-\ell+1)$.

We thus have:

$$
\operatorname{Poin}\left(\mathcal{R}_{\mathscr{X}}^{1}, x\right)=x^{-d+\ell-1} \operatorname{Poin}\left(\overline{\mathcal{I}_{\mathscr{Y}}}, x\right) .
$$

We can now compute the $\Psi$-function associated with the modules of logarithmic residues:

$$
\begin{aligned}
\Psi\left(\mathcal{R}_{\mathscr{X}}^{\bullet}, x, t\right) & =\operatorname{Poin}\left(\mathcal{R}_{\mathscr{X}}, x\right)+(t(1-x)-1) \operatorname{Poin}\left(\mathcal{R}_{\mathscr{X}}^{1}, x\right) \\
& =1+x^{\ell-d} \operatorname{Poin}\left(\overline{\mathcal{I}_{\mathscr{Y}}}, x\right)+(t(1-x)-1) x^{\ell-d-1} \operatorname{Poin}\left(\overline{\mathcal{I}_{\mathscr{Y}}}, x\right) \\
& =1+x^{\ell-d-1} \operatorname{Poin}\left(\overline{\mathcal{I}_{\mathscr{Y}}}, x\right)(x+t(1-x)-1) \\
& =1+x^{\ell-d-1} \operatorname{Poin}\left(\overline{\mathcal{I}_{\mathscr{Y}}}, x\right)(t-1)(1-x)
\end{aligned}
$$

Therefore, $\Psi\left(\mathcal{R}_{\mathscr{X}}^{\bullet}, x, 1\right)=1$, which gives us corollary 5.1 .

Remark 5.4. The computation of $\mathcal{R}_{\mathscr{X}}$ made in the proof of corollary 5.1 is not specific to line arrangements. Let $C \subseteq\left(\mathbb{C}^{\ell}, 0\right)$ be a reduced singular complete intersection curve which is quasihomogeneous with respect to the weights $\left(w_{1}, \ldots, w_{\ell}\right)$. Let $c_{1}, \ldots, c_{\ell} \in \mathbb{C}$ be such that $g=\sum_{i=1}^{\ell} c_{i} J_{i}$ and $y=\sum_{i=1}^{\ell}(-1)^{i-1} c_{i} w_{i} x_{i}$ induce non zero divisors in $\mathcal{O}_{C}$. By [Pol16b, Proposition 6.1.24], the module $\mathcal{R}_{C}$ is generated by 1 and $\frac{y}{g}$. Then with exactly the same proof as for corollary 5.1 , one can prove that for any equidimensional reduced singular subspace $X \subseteq C$ of dimension 1, there exists $\rho_{1}=a_{1} \frac{y}{g}+c_{1} \in \mathcal{R}_{X}$ with $c_{1} \neq 0$ and that the module of logarithmic multi-residues $\mathcal{R}_{X}$ is generated by the elements in $\mathcal{I}_{Y} \frac{y}{g}$ and $\rho_{1}$, where $Y$ denotes the union of the components of $C$ which are not in $X$.

5.2. Higher dimensional subspace arrangements. The question which then arises is to determine if the condition $\Psi\left(\mathcal{R}_{\mathscr{X}}^{\bullet}, 1,1\right)=1$ is satisfied for any equidimensional subspace arrangement of any dimension. The answer is no, as it is shown by the following example.

Example 5.5. Let us consider $\mathcal{I}_{\mathscr{X}}=\langle x, z\rangle \cap\langle y, t\rangle=\langle x y, x t, y z, z t\rangle$. A reduced complete intersection subspace arrangement containing $\mathscr{X}$ is given by the ideal $\mathcal{I}_{\mathscr{C}}=\langle x y, z t\rangle$. We set $h_{1}=x y, h_{2}=z t$, $h=x y z t$. Let $\mathcal{I}_{\mathscr{Y}}=\langle x y, x z, y t, z t\rangle$ be the radical ideal defining the union of the irreducible components of $\mathscr{C}$ which are not contained in $\mathscr{X}$. 
Let us compute the module of multi-logarithmic differential forms. We have:

$$
\Omega^{0}(\log \mathscr{X} / \mathscr{C})=\frac{1}{h} \mathcal{I}_{\mathscr{C}}, \quad \Omega^{1}(\log \mathscr{X} / \mathscr{C})=\frac{1}{h} \mathcal{I}_{\mathscr{C}} \Omega^{1}, \quad \Omega^{4}(\log \mathscr{X} / \mathscr{C})=\frac{1}{h} \mathcal{I}_{\mathscr{Y}} \Omega^{4} .
$$

Computations made with Singular gives that

$$
\frac{1}{h}\left(a_{1} \mathrm{~d} z \wedge \mathrm{d} t+a_{2} \mathrm{~d} y \wedge \mathrm{d} t+a_{3} \mathrm{~d} y \wedge \mathrm{d} z+a_{4} \mathrm{~d} x \wedge \mathrm{d} t+a_{5} \mathrm{~d} x \wedge \mathrm{d} z+a_{6} \mathrm{~d} x \wedge \mathrm{d} y\right) \in \Omega^{2}(\log \mathscr{X} / \mathscr{C})
$$

if and only if

$$
a_{1}, a_{3}, a_{4}, a_{6} \in \mathcal{I}_{\mathscr{C}}, a_{2} \in\langle x z, x y, z t\rangle, a_{5} \in\langle y t, x y, z t\rangle .
$$

In addition, we have:

$$
\frac{1}{h}\left(a_{1} \mathrm{~d} y \wedge \mathrm{d} z \wedge \mathrm{d} t+a_{2} \mathrm{~d} x \wedge \mathrm{d} z \wedge \mathrm{d} t+a_{3} \mathrm{~d} x \wedge \mathrm{d} y \wedge \mathrm{d} t+a_{4} \mathrm{~d} x \wedge \mathrm{d} y \wedge \mathrm{d} z\right) \in \Omega^{3}(\log \mathscr{X} / \mathscr{C})
$$

if and only if

$$
a_{1}, a_{3} \in\langle x z, x y, z t\rangle, a_{2}, a_{4} \in\langle y t, x z, z t\rangle .
$$

Free resolutions of these modules are given by:

$$
\begin{aligned}
& 0 \leftarrow \Omega^{0}(\log \mathscr{X} / \mathscr{C}) \leftarrow S(2)^{2} \leftarrow S(0) \leftarrow 0 \\
& 0 \leftarrow \Omega^{1}(\log \mathscr{X} / \mathscr{C}) \leftarrow S(2)^{4} \leftarrow S(0)^{2} \leftarrow 0 \\
& 0 \leftarrow \Omega^{2}(\log \mathscr{X} / \mathscr{C}) \leftarrow S(2)^{14} \leftarrow S(0)^{4} \oplus S(1)^{4} \leftarrow 0 \\
& 0 \leftarrow \Omega^{3}(\log \mathscr{X} / \mathscr{C}) \leftarrow S(2)^{12} \leftarrow S(1)^{8} \leftarrow 0 \\
& 0 \leftarrow \Omega^{4}(\log \mathscr{X} / \mathscr{C}) \leftarrow S(2)^{4} \leftarrow S(1)^{4} \leftarrow S(0) \leftarrow 0
\end{aligned}
$$

It is then possible to compute that

$$
\Psi\left(\Omega^{\bullet}(\log \mathscr{X} / \mathscr{C}), x, t\right)=\frac{1}{x^{4}}\left(x^{4} t^{4}-4 x^{3} t^{4}+4 x^{3} t^{3}+4 x^{2} t^{4}-4 x^{2} t^{3}+2 x^{2} t^{2}\right) .
$$

In particular, $\Psi\left(\Omega^{\bullet}(\log \mathscr{X} / \mathscr{C}), 1, t\right)=t^{4}+2 t^{2}$ and $t^{4}-\Psi\left(\mathcal{R}_{\mathscr{X}}^{\bullet}, 1, t\right)=t^{4}-2 t^{2}$ whereas the characteristic polynomial is $\chi(\mathscr{X}, t)=t^{4}-2 t^{2}+1$. In particular, $\Psi\left(\mathcal{R}_{\mathscr{X}}^{\bullet}, 1,1\right)=2$.

However, there exist subspace arrangements of dimension greater than one such that the relation $t^{\ell}-\Psi\left(\mathcal{R}_{\mathscr{X}}^{\bullet}, 1, t\right)=\chi(\mathscr{X}, t)$ is satisfied, for example the complete intersection of $\mathbb{C}^{4}$ defined by $\langle x y, z t\rangle$. An interesting problem would be to characterize the subspace arrangements for which the generalized Solomon-Terao formula holds.

\section{REFERENCES}

[Ale12] Alexandr G. Aleksandrov. Multidimensional residue theory and the logarithmic de Rham complex. $J$. Singul., 5:1-18, 2012.

[Ale14] Alexandr G. Aleksandrov. Residues of logarithmic differential forms in complex analysis and geometry. Anal. Theory Appl., 30(1):34-50, 2014.

[AMMN18] Takuro Abe, Toshiaki Maeno, Satoshi Murai, and Yasuhide Numata. Solomon-terao algebra of hyperplane arrangements. ArXiv.org, (arXiv:1802.04056v1), 2018.

[AT01] Aleksandr G. Aleksandrov and Avgust K. Tsikh. Théorie des résidus de Leray et formes de Barlet sur une intersection complète singulière. C. R. Acad. Sci. Paris Sér. I Math., 333(11):973-978, 2001.

[Ath96] Christos A. Athanasiadis. Characteristic polynomials of subspace arrangements and finite fields. Adv. Math., 122(2):193-233, 1996.

[Bjo94] Anders Bjorner. Subspace arrangements. In First European Congress of Mathematics, Vol. I (Paris, 1992), volume 119 of Progr. Math., pages 321-370. Birkhäuser, Basel, 1994.

[Ker83] Masumi Kersken. Cousinkomplex und Nennersysteme. Math. Z., 182(3):389-402, 1983.

[Ker84] Masumi Kersken. Reguläre Differentialformen. Manuscripta Math., 46(1-3):1-25, 1984.

[LNTY17] Ye Liu, Tan Nhat Tran, and Masahiko Yoshinaga. $g$-tutte polynomials and abelian lie groups arrangements. ArXiv.org, (arXiv:1707.04551), 2017.

[OT92] Peter Orlik and Hiroaki Terao. Arrangements of hyperplanes, volume 300 of Grundlehren der Mathematischen Wissenschaften [Fundamental Principles of Mathematical Sciences]. Springer-Verlag, Berlin, 1992.

[Pol16a] Delphine Pol. Characterizations of freeness for Cohen-Macaulay spaces. ArXiv.org, (arXiv:1512.06778v2), 2016. 
[Pol16b] Delphine Pol. Singularités libres, formes et résidus logarithmiques. Thèse de doctorat, (tel-01441450), 2016.

[Pol17] Delphine Pol. On the values of logarithmic residues along curves. to appear at Annales de l'Institut Fourier, (arXiv:1410.2126v4), 2017.

[Sai80] Kyoji Saito. Theory of logarithmic differential forms and logarithmic vector fields. J. Fac. Sci. Univ. Tokyo Sect. IA Math., 27(2):265-291, 1980.

[ST87] L. Solomon and H. Terao. A formula for the characteristic polynomial of an arrangement. Adv. in Math., 64(3):305-325, 1987.

Delphine Pol, Department of Mathematics, Hokkaido University, Kita 10, Nishi 8, Kita-Ku, Sapporo 060-0810, JAPAN

E-mail address: pol@math.sci.hokudai.ac.jp 